\title{
Product design lifecycle information model (PDLIM)
}

\author{
Xiaojing Niu ${ }^{1} \cdot$ Meili Wang ${ }^{2} \cdot$ Shengfeng Qin $^{1}$ (D) \\ Received: 7 January 2021 / Accepted: 21 August 2021 / Published online: 28 September 2021 \\ (C) The Author(s) 2021
}

\begin{abstract}
In response to rapidly changing market and customer needs, product design and development (PDD) is evolving into a humancentred and data-driven design paradigm. The design environment gets more open often involving crowdsourcing and the design process becomes more complex, considering product family design along product whole lifecycle development, and needing more data support. Therefore, it is critical to effectively capture, share, and manage design-related information in such a complex design environment. From this perspective, it is a prerequisite to have a proper product design lifecycle information model (PDLIM) to guide information gathering, sharing and management. To the best of our knowledge, currently, there lacks such a PDLIM to support effective PDD, though digital twin (DT) technology shows a great potential of supporting product lifecycle information collection and management. In this paper, the overall structure of the proposed PDLIM is firstly developed to frame in all main product lifecycle stages and the corresponding key phases for structurally capturing and storing necessary data along a product lifecycle. Secondly, key design information items against the main product lifecycle stages and their corresponding key phases are explored from literature reviews and case study analyses. Thirdly, the necessity of the identified information items in the PDLIM is qualitatively evaluated by two case studies. Finally, the PDLIM is further evaluated by applying formal object-role modelling (ORM) to demonstrate how design information items are used and interacted in exemplary design interaction scenarios, and to approve that it can be formally described and managed as an information model. The evaluation results show that the PDLIM is feasible to be adapted in a crowdsourcing-combined PDD process for supporting design management, reviewing, quality control, and next round product redesign and improvement.
\end{abstract}

Keywords Product design and development process, $\cdot$ Lifecycle, $\cdot$ Information model, $\cdot$ Crowdsourcing, $\cdot$ Digital twin

\section{Introduction}

With customer needs and market demands continually evolving and shifting over time, small and medium enterprises (SMEs) in manufacturing face a great demand for a variety of new products in a short time $[1,2]$. In response to this challenging demand, as effective ways to ensure the competitivity of SMEs, there are three main product design trends, which require a great design information support.

The first trend is product family design (PFD) $[1,2]$ to realize mass customization in manufacturing, which plays a significant role in rapid product development mainly by

Shengfeng Qin

sheng-feng.qin@northumbria.ac.uk

1 School of Design, Northumbria University, Newcastle upon Tyne NE1 8ST, UK

2 College of Information Engineering, Northwest A\&F University, Yangling 712100, Shaanxi, China sharing product development assets and various feedback on existing products in use. It increases a product's variants by sharing assets such as components, modules, processes, and knowledge across the products, not only satisfying a variety of market segments but also helping SMEs gain an edge in fierce market competition through reduced product development cost and time. The PFD is a holistic product design approach requiring strong support of data and information sharing along the product lifecycle [1]. However, different stages of a PFD in different generations [1] are normally conduced separately by different departmental teams or collaborators. As a result, the design process crossing several product generations is broken into fragments and the related design information set is usually proprietary to certain collaborators on the supply chain $[3,4]$. The lack of continuity and coherence of recorded key product design data and information through the product family lifecycle is a big barrier for next generation product design [2]. This in turn requires a robust product design information model to guide the collection and recording of the product design data and information by considering all design 
stages holistically through its family lifecycle. The lack of such an information model to support this requirement is the key barrier stopping PFD from being widely implemented by SMEs in practice.

The second trend is crowdsourcing-based product design and development (C-PDD) [5, 6], which enables SMEs to directly outsource design tasks to the Internet connected crowds during their PDD processes. This can help SMEs transform product design from "design for customers" to "design by customers" and "design with customers" in an opener way to reduce the product development time by flexibly increasing their human resources and capability from the crowds. Currently, the crowdsourced design activities are supported by a combined design environment of traditional inhouse design and platform-based crowdsourcing design. Effective design information communication and datainformed decision making in this combined environment are key influential factors for good design quality [7]. Although crowdsourcing opens up opportunities for flexibly coordinating external participants or even experts/professionals for knowledge intensive product design activities, it also brings some concerns on product design quality and Intellectual Properties [8]. In the last decade, with the adoption of collaboration strategy nationally and internationally, the PDD process has become progressively more complex because of the dynamics of design participants and their skills, the unpredictable outcomes at each design stage, and the lack of quality control monitoring measures on the crowdsourcing PDD process. As a result, crowdsourcing has not been widely adopted in manufacturing practices. Furthermore, crowdsourcing PDD also faces the problem of fragmented design information as in PFD. According to the board of innovation (https://www. boardofinnovation.com/staff_picks/open-innovationcrowdsourcing-resources/), crowdsourcing has been demonstrated to be effective in data collection, problem solving, and collective intelligence, but little research has been investigated on how to ensure an iterative PDD process in a combined design environment. To address this, a proper product design information model that can describe product design information throughout the product (family) lifecycle is required.

The third one is digital twin-driven product design and development (DT-PDD) [4, 9]. Underpinned by Internet of Things (IoT), Big Data, and Artificial Intelligence, etc., DT enables all physical components in a product lifecycle to map to their virtual twins which can capture and analyze various product performance and usage data in real-time and adjust the physical twins automatically and timely. These captured product performance data and information on the existing products in turn can support product family design in the next generation by addressing design issues embedded in the existing products. Owing to its advantages of high-fidelity simulation and cyber-physical interconnectivity empowered by information and communication technology, DT provides a new way of information collection for accelerating datadriven PDD and a new way of information communication especially at product use and maintenance stages. It is recognized that DT can be a trend-setter for many industry sectors [10]. Currently, DT is mainly implemented at a later PDD stage, especially product manufacturing $(35 \%)$, or product health management $(38 \%)$ at product use and maintenance stages $[11,12]$. Its potential support to earlier stages of a PDD process is also being explored [13]. In addition, DT is also applied to monitor the usage conditions of a complex product family [14]. However, the research on DT is still at a very early stage. Although a lot of work has been done in each of these stages in isolation, there still lacks a consistent product design information model to connect these stages for improving a product design in a holistic manner.

In order to support the three PDD trends with existing crowdsourcing and DT technologies, SMEs still need a great support of all design information along a product lifecycle to enable them to learn from their previous design projects, and their past and existing products' usage data [1] in their product families. In this regard, design information is mainly generated from current product design and development activities, past and existing products' usage data over their later product lifecycle, and previous generational product design information. Design information is also in many different forms such as $2 \mathrm{D}$ drawing, 3D model, etc. generated from various design tools and information capturing methods. To effectively support the above design trends, product usage data are required to be acquired and analyzed in a real-time manner and all design information over the product lifecycle is required to be seamlessly captured, stored, and retrieved for information sharing and communication among all involved departmental teams and crowds in combined design environments. This in turn requires a product design lifecycle information model to support effective design information identification, capturing, and use. Due to the widespread collaborative product design and development activities among various design teams both inside and outside SMEs and the complexity of design information itself, currently, there is no existing product design information model that can fully support this requirement, especially at the early stages of PDD.

So far, existing studies in the related fields are mainly limited on product model. In a conventional design environment, to reduce the iteration of product design and manufacturing in a PDD process, Standard for the Exchange of Product Model Data (STEP), Core Product Model (CPM) and its extended Open Assembly Model, Design-Analysis Integration Model, and Product Family Evolution Model [15] have been proposed for design data exchanges among different CAx ( $\mathrm{x}$ means design, engineering, and manufacturing) systems. The information captured by these models is mainly related to product geometrical features, structures, materials, 
manufacturing methods, and assembly relationships, etc. The detailed information supported by these models is described in Section 3.2. While the relevant information beyond the specified formats such as markets' needs, and customers' needs/ desires identified at pre-design stage and user feedback generated during and after the product manufacturing stage is excluded. The lack of this design information will result in the discontinuity of design information in the product design lifecycle, affecting design decision-making and improvement in an iterative design process. In addition, a product generated real performance data (most likely differing from the simulated design data) during the product in-use stage is also excluded, making it hard to monitor the product real-time performance and predict required maintenance based on that. To our knowledge, these existing models are unable to support a holistic product design and development process requiring necessary data/information support through the product whole lifecycle. From this aspect, a PDLIM covering design information from marketing to disposal is needed.

In the same vein, many research efforts have been put into design information management by Product Data Management (PDM) and Product Lifecycle Management (PLM) systems, expected to provide insights of product development to ensure a timely and on-budget delivery of highquality and competitive products. The former focuses on product (design) version management, aiming to maintain the consistency across versions and track each change as new versions come in, while the latter aims to manage the change processes and workflows that drive product development. To better aid PDD processes, many SMEs have expanded PDM systems to PLM ones. However, these systems rely heavily on participants along a product lifecycle to collect information and then record it in a manual way, possibly leading to the loss of important decision-making process and information in the previous design projects. Not knowing adequate information about the prior design projects could lead to difficulty in being understood by future projects [13]. In addition, the information managed by PDM/PLM systems does not cover a product's real-time status data, its operating environment (or use scenario) and interactions with customers at product in-use stage. But now, the emergence of DT technology makes it possible to collect these data and use them for next round product design and development [16]. Meanwhile, crowdsourcing technology provides a flexible way to collect information in a product design and development process, such as the information about product application environment and user experience data [17]. These technologies enable the continuity of design information by providing new ways to collect it throughout the product lifecycle. It is believed that the information generated and collected during the product lifecycle is valuable in assisting the next round (generation) of product design and development.
Different from the exiting product information exchange models that support design information communication among computer-aided tools and between late design stages and the manufacturing stage, the proposed PDLIM is required to share design information at much higher levels of abstraction between any two different stages in the whole product lifecycle. Furthermore, where the design information generates from and how it is collected, processed, analyzed, and modelled are also required to be addressed in the PDLIM. As the data/information collection, analysis, and modelling have been relatively well studied [18, 19], this paper mainly focuses on what information should be collected in the product lifecycle in in-house and crowdsourcing combined design environments. The PDLIM forms the basis for ongoing collection and synthesis of design information in a product lifecycle.

Although such an information model for product design and development over the lifecycle is very useful, the research on this topic is very few [20]. The reason for that may be because it requires an advanced digital manufacturing platform support. But now, the emergence of DT platform for manufacturing makes this information available for collection and reuse in a closed-loop of product lifecycle development [16].

The proposed PDLIM can be reviewed as an information wardrobe with a structure and many drawers. Each drawer can have a label to describe a key design information item at a higher level of abstraction (class level) and have an inner space for incrementally storing a varied number of detailed information and data entities over the product lifecycle and product family evolution periods in various formats such as 3D models, drawings, sketches, graphs, and worksheets. In the PDLIM, not all information items are required in a specific product design, just like in a wardrobe, not all drawers are used fully all the time, but the PDLIM provides a design information wardrobe containing all designed drawers to possibly store the classified key information items in a product lifecycle as much as possible.

Such a product design information model could help designers identify the information required to make decisions along the PDD lifecycle by providing an information structure for design information acquisition, storage, retrieval, representation, communication, and exchange among designers and other stakeholders. It enables better understanding of and insights into the course of the process [20]. We believe that this PDLIM is critical in supporting the above three emerging PDD trends. It is a prerequisite for practising a holistic and sustainable design process through effective integration with other key product lifecycle development stages.

The novelties of this research are as follows:

(1) A new research perspective under the DT, crowdsourcing and product family development trends 
for developing a new PDLIM to support the new closedloop product family lifecycle design and development.

(2) The proposed PDLIM goes beyond the existing product model and product lifecycle management model and enables their integration.

(3) The proposed PDLIM is an information wardrobe and a technological enabler for capturing, recording, and reusing not only the final product design result data/information, but also product design process, product use and maintenance, and decision-making information during the product lifecycle.

The specific research contribution is threefold:

(1) The proposed PDLIM can support PDD in both traditional and crowdsourcing-based collaborative design environments, making design opener with flexible crowd resource.

(2) The PDLIM provides an overall product design information wardrobe structure to support not only product lifecycle design but also product family design and development, bettering lifecycle design information sharing, and thus improving decision-making and design quality.

(3) The PDLIM can support the new data-informed product design paradigm enabled by DT technology, supporting smart manufacturing.

\section{Research methodology}

Product information model plays an important role in minimising knowledge gap between two different design stages, facilitating knowledge exchange, retrieval, and reuse during the PDD process and accelerating data-driven PDD $[21,22]$. The leading approach for representing product data is feature-based product modelling which focuses on how to model design information with generic modelling terms in terms of design ontologies [21] and the mapping of design and manufacturing ontologies [22] rather than what information items should be modelled at each design phase. Although STEP and CPM indicate many important information items, they fail to support the whole PDD lifecycle. To complement STEP and CPM to support PDD in an increasingly complex design environment, many studies have been conducted to collect and model additional product design information that is not covered by STEP and CPM from the perspective of product lifecycle. For example, Song et al. [23] proposed a four-dimensional view model to manage product simulation data in virtual prototype systems. However, the design information is still not managed in a continuous and holistic way.
Therefore, a systematic research approach with six steps is adopted in our research (see Fig. 1) to develop the PDLIM to support the main design trends in crowdsourcing-combined design environments. In Step 1, we first develop the overall structure of the PDLIM based on the five key product design and development stages (see Fig. 2), which accommodates high-level design information along a product lifecycle as much as possible in a structural way. Second, we develop key information exploration guides for each design phase including (1) what information is needed and where it is generated, (2) how and when the information needs to be communicated for information sharing, (3) how the information is used in design reviewing and decision-making, (4) what the design team structure and key players are in the design process, and (5) how the information could be updated with a history/version tracing for up-to-date applications. In Step 2, we explore typical product design phases against each design stage. In Steps 3 and 4, we explore typical design actions in each design phase, which may involve different design teams working in both in-house and crowdsourcing design environments in action and their interactions. In Step 5, we apply the key information exploration guides to explore typical design information items over the developed structure to be accommodated in the PDLIM and evaluate the PDLIM with case studies. And in Step 6, we evaluate the relationships of identified information items in the PDLIM and their interaction and manageability by ORM modelling method. The corresponding research methods for each step is also shown in Fig. 1. This research process could be iterative.

\section{Development of the PDLIM}

This section mainly presents the first four development steps in detail. The other two steps are described in due sections.

\subsection{The overall structure of PDLIM}

The overall structure of the PDLIM is shown in Fig. 2 as a multi-layered information model structure. It represents the information model at the system level, mainly illustrating product lifecycle design processes and information structure for supporting the closed-loop product design along a product lifecycle. In the closed-loop PDD, the design lifecycle information model is supported by its physical part and virtual part. In the physical part, primary design information is generated by real-world design processes and interactions among design teams in certain design environments, as well as product usage and product-user interaction data. The generated design information and process information in the physical world are collected and then processed before being uploaded to the information repositories in the virtual part. In the virtual part, all stored information can be further analyzed, simulated, 
Fig. 1 The systematic research approach in this research

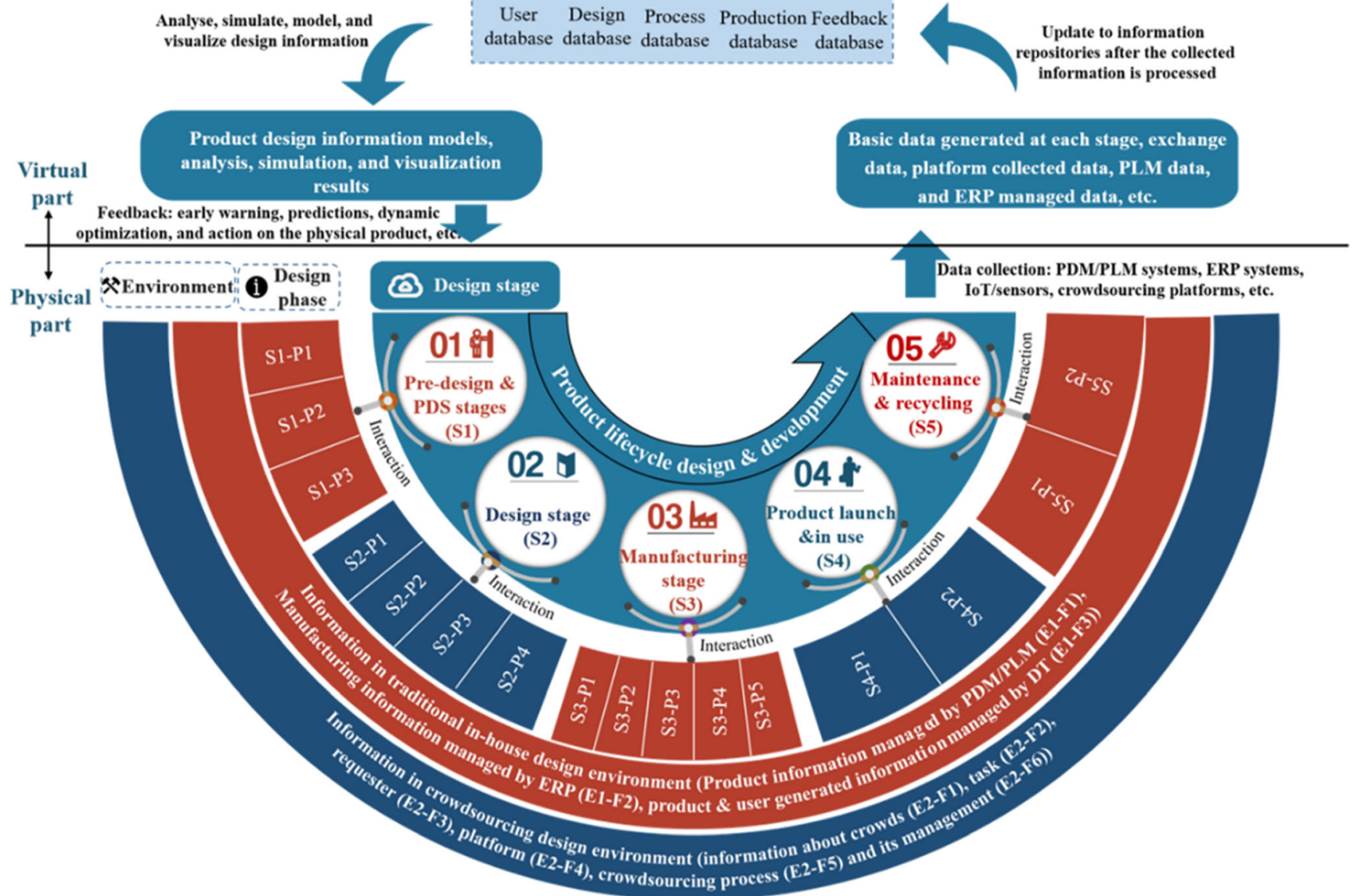

\section{Research process}

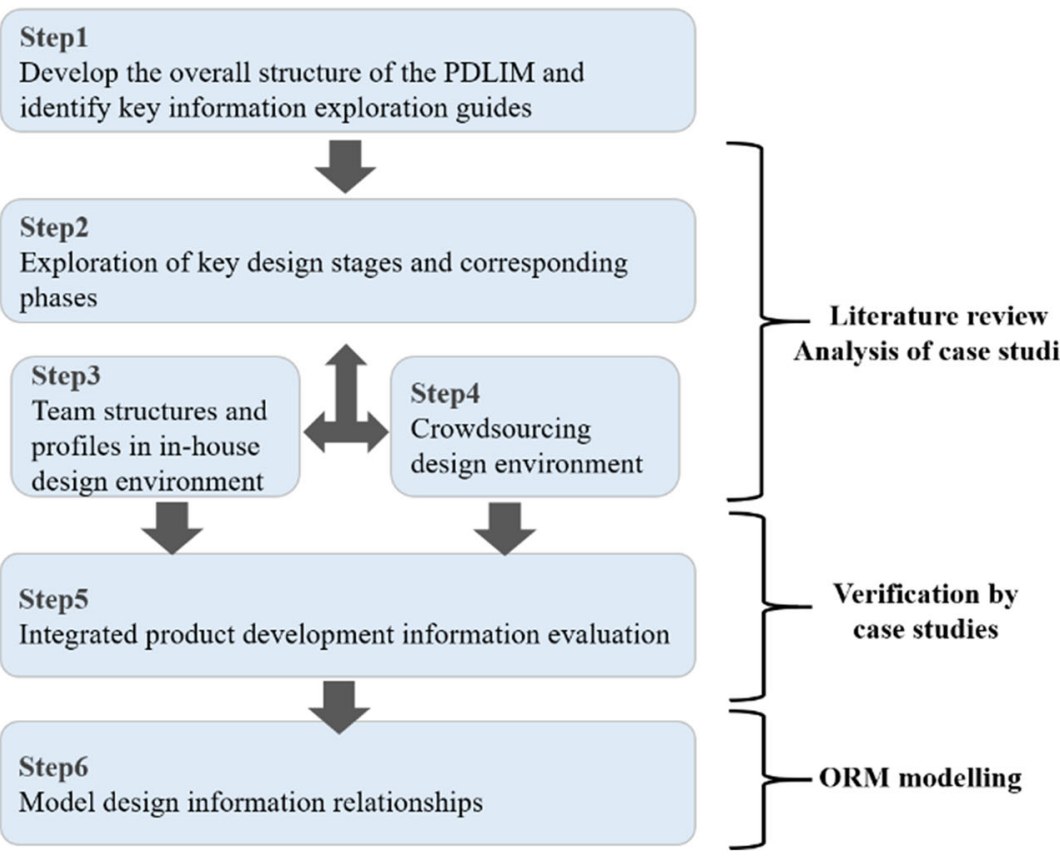

Research methods

Literature review Analysis of case studies profiles in in-house design environment

Step5

Integrated product development information evaluation

Step6

Model design information relationships

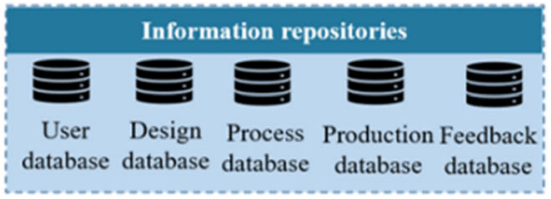

Basic data generated at each stage, exchange data, platform collected data, PLM data, and ERP managed data, etc.

Fig. 2 The overall structure of the PDLIM 
modelled, and visualized to generate secondary data and information like various simulation models in DTs for feedback to next product design loop (either revised design or new generation design). For example, early product failure warning or maintenance predictions for smart maintenance servicing and smart product reconfiguration.

In this model, the PDD process in the physical part is structured into five typical lifecycle design stages [24]: pre-design $\&$ product design specification (PDS) stage (S1), design stage (S2), manufacturing stage (S3), product launch \& in use stage (S4), and maintenance \& recycling stage (S5). Among these stages, the pre-design and design stages account for 70\%-80\% of the product quality [25]. To reduce design iterations, 'Design for X' (X means manufacturability, reusability, safety, etc.) techniques are usually considered at the pre-design and design stages to optimize product designs [26]. These five key design stages are presented in the inner "Design stage" layer from the left to right indicating a product lifecycle design and development.

Next, key design phases associated with each key stage are identified from literature study [24, 27]. As a result, three phases related to product design research are identified for S1; S2 involves four phases covering conceptual and detail design; S3 has five phases in product manufacturing; S4 contains two phases about product lunch and product in use; and S5 has two phases linking to product maintenance and recycling. The information at each design phase is presented in the "Design phase" layer. The details of specific phases at each stage are shown in Fig. 3.

Finally, the design environment information is presented on the "Environment" layer. It includes two sub-layers. One is the traditional in-house design environment consisting of design teams, design/manufacturing management tools such as PDM/PLM/ERP systems [27, 28] and manufacturing platforms such as DTs. This sub-layer represents the information generated from human players, products, and other tools. The other sub-layer is to represent a crowdsourcing environment related data/information covering tasks, crowds, requesters, platforms, crowdsourcing processes, and information management.

The top part of Fig. 2 also illustrates how the information in the PDLIM could be stored in information repositories with integration to $\mathrm{PDM} / \mathrm{PLM} / \mathrm{ERP}$ systems, DT, and crowdsourcing for supporting the next round of datainformed product design and development in a closed-loop fashion. As for the database implementation techniques, in order to support a variety of data formats described in the PDLIM, it is more suitable to apply MongoDB [29] (a NoSQL database that stores data in the form of "key-value pairs") to store a very large amount of data with various formats, since MongoDB is a document-oriented database technology that enables flexible data models, high availability and horizontal scalability in applications.

Between the "Design stage" layer and the "Design phase" layer, it is the "Interaction" information layer. The information on this layer is secondary information capturing how primary information on the three main information layers are interacted and utilized for supporting design activities and decision making. In Section 5, we will demonstrate how information interacted with each other in typical application scenarios along the product design and development lifecycle, which could be regarded as design activity-based information.

\subsection{Key design information exploration}

Based on Figs. 2 and 3, for each key stage, key design information items at each phase are explored to enrich the information model. Note that the identified key design information items are commonly used at each phase as design space exploration guides for designers to extract the corresponding information values (data entities) for later communication

\begin{tabular}{|c|c|c|c|c|}
\hline $\begin{array}{c}\text { Pre-design \& PDS } \\
\text { stage (S1) }\end{array}$ & Design stage (S2) & $\begin{array}{l}\text { Manufacturing } \\
\text { stage (S3) }\end{array}$ & $\begin{array}{c}\text { Launch } \& \text { in use } \\
\text { stage (S4) }\end{array}$ & $\begin{array}{c}\text { Maintenance } \& \\
\text { recycling stage (S5) }\end{array}$ \\
\hline $\begin{array}{l}\text { Market needs analysis } \\
\& \text { evaluation (S1-P1) }\end{array}$ & $\begin{array}{c}\text { Design concept } \\
\text { generation }(\mathrm{S} 2-\mathrm{P} 1)\end{array}$ & $\begin{array}{c}\text { Manufacturing } \\
\text { scheduling (S3-P1) }\end{array}$ & Product launch (S4-P1) & $\begin{array}{l}\text { Product maintenance } \\
\text { (S5-P1) }\end{array}$ \\
\hline $\begin{array}{c}\text { User needs analysis \& } \\
\text { evaluation (S1-P2) }\end{array}$ & $\begin{array}{l}\text { Concept development } \\
\text { and evaluation (S2-P2) }\end{array}$ & $\begin{array}{c}\text { Actual product parts } \\
\text { manufacturing (S3-P2) }\end{array}$ & Product in use (S4-P2) & $\begin{array}{l}\text { Product recycling } \\
\text { (S5-P2) }\end{array}$ \\
\hline \multirow[t]{3}{*}{$\begin{array}{c}\text { Product design } \\
\text { specification (S1-P3) }\end{array}$} & $\begin{array}{c}\text { Detail design and } \\
\text { evaluation (S2-P3) }\end{array}$ & $\begin{array}{c}\text { Product assembly } \\
\text { (S3-P3) }\end{array}$ & & \\
\hline & $\begin{array}{c}\text { Integrated service } \\
\text { development (S2-P4) }\end{array}$ & $\begin{array}{l}\text { Product testing and } \\
\text { evaluation (S3-P4) }\end{array}$ & & \\
\hline & & $\begin{array}{c}\text { Product transportation } \\
\text { (S3-P5) }\end{array}$ & & \\
\hline
\end{tabular}

Fig. 3 Key phases at each design stage of a PDD process 
with collaborators. The identified key information items are adaptable for a specific product design application case.

With the information exploration guidelines (stated in Section 2), we first collected 51 case studies related to product or service design projects, which were performed by undergraduates on the Northumbria School of Design's BA Design for Industry course. And then we extracted key design information items at product pre-design and design stages. After analysing them, design studies [24, 30,31] were also reviewed to enrich design information at these two design stages. As a result, the key design information items at phases S1 and S2 are identified and presented in Fig. 4.

In $\mathrm{S} 2-\mathrm{P} 3$, the $\mathrm{CAx}$ models refer to various $\mathrm{CAD} / \mathrm{CAE} /$ CAM models [32, 33]. To improve product design and development efficiency, these CAx models [34] are required to exchange data with each other supported by existing product data exchange models especially STEP and CPM as well as its extensions [15]. The detailed information items listed in Table 1 is embedded in CAx models and can be shared and exchanged among them $[35,36]$.

At the pre-design and design stages, design failures are usually created unintentionally. Hence, to ensure all design requirements have been completely met and the potential design problems have been addressed before manufacturing, design failure mode and effects analysis (DFMEA) [37] from the prototype control plan and verification tests is usually adopted as a qualitative tool to review product designs by identifying potential failures, potential effects caused by failures, and potential causes for failures, etc. In the DFMEA process, a standard DFMEA worksheet that treats function as the smallest unit of DFMEA analysis is used as a tool to continuously document design changes and the corresponding caused quality problems, enabling the product reliability and safety in the product lifecycle. DFMEA analysis focuses on not only the part/sub-system design but also the interface design between parts/sub-systems, which are represented by assembly features. The documented information is helpful in enabling product performance, reliability, and safety in due courses [37]. In the PDLIM, the information documented in the DFMEA worksheets in the detail design and evaluation (S2P3) phase can well accommodate testing and evaluation criteria, potential failures, potential effects caused by failures, potential causes for failures, severity level, and suggested actions/countermeasures. At the end of the design evaluation phase, a design verification report from the stakeholders' reviews covering the test items, criteria, and procedures, etc. could be generated for the identified information items. The design verification report will determine the product development direction, either going back to redesign the product if severe problems exist in the current design or entering the next design phase where the identified problems could be compensated by value-added services.

When the product design is verified, the PDD process enters the manufacturing stage (S3), in which the key information items are mainly identified from design studies [4, 20, 38], as shown in Fig. 5.

In the product manufacturing stage (S3), control plans at the manufacturing scheduling phase (S3-P1) and manufacturing technology and process quality checking in the actual parts manufacturing phase (S3-P2) involve process failure mode and effects analysis (PFMEA). PFMEA is crucial in process risk identification, assessment, mitigation, and control [39]. PFMEA looks at each step of the PDD process to identify risks and possible failures from man, methods, material,

\begin{tabular}{|c|c|c|c|c|c|c|c|}
\hline $\begin{array}{l}\text { Design } \\
\text { Phase }\end{array}$ & $\begin{array}{l}\text { Market needs analysis } \\
\text { \& evaluation (S1-P1) }\end{array}$ & $\begin{array}{l}\text { User needs analysis } \\
\text { \& evaluation (S1-P2) }\end{array}$ & $\begin{array}{c}\text { Product design } \\
\text { specification (S1-P3) }\end{array}$ & $\begin{array}{l}\text { Design concept } \\
\text { generation ( } \mathbf{S 2} 2-\mathrm{P} 1)\end{array}$ & $\begin{array}{l}\text { Concept development } \\
\text { \& evaluation (S2-P2) }\end{array}$ & $\begin{array}{l}\text { Detail design and } \\
\text { evaluation (S2-P3) }\end{array}$ & $\begin{array}{c}\text { Integrated service } \\
\text { development (S2-P4) }\end{array}$ \\
\hline $\begin{array}{c}\text { Key } \\
\text { information } \\
\text { items }\end{array}$ & $\begin{array}{l}\text { 1. Competitor products } \\
\text { 2. Development/market } \\
\text { trends } \\
\text { 3. Marketing strategies } \\
\text { of competitors } \\
\text { 4. Packaging } \\
\text { 5. Market potential } \\
\text { 6. Product positioning } \\
\text { 7. Targeting strategy } \\
\text { 8. Market share } \\
\text { 9. Marketing context } \\
\text { 10. Market sales } \\
\text { 11. Prince point analysis } \\
\text { 12. Innovation strategy } \\
\text { 13. Brand audit } \\
\text { 14. Competitive } \\
\text { advantage } \\
\text { 15. Unique selling point } \\
\text { 16. Feasibility study, } \\
\text { etc. }\end{array}$ & $\begin{array}{l}\text { 1. Customer segments } \\
\text { 2. Customer motivations } \\
\text { 3. Customer personas } \\
\text { 4. Frustrations/pain } \\
\text { points for each customer } \\
\text { segment } \\
\text { 5. Customer journey } \\
\text { maps } \\
\text { 6. Customer experience } \\
\text { 7. Customer comments } \\
\text { on the product } \\
\text { 8. Human context } \\
\text { 9. Stakeholders } \\
\text { 10. Stakeholders' } \\
\text { comments on the product } \\
\text { 11. Customer preferences } \\
\text { 12. Observation study } \\
\text { 13. Emotional design } \\
\text { 14. Expert interviews, } \\
\text { etc. }\end{array}$ & $\begin{array}{l}\text { 1. Product size \& weight } \\
\text { 2. Performance } \\
\text { requirements } \\
\text { 3. Service environment } \\
\text { 4. Safety requirements } \\
\text { 5. Reliability standards } \\
\text { \& requirements } \\
\text { 6. Ergonomic } \\
\text { requirements } \\
\text { 7. Product aesthetics } \\
\text { 8. Maintenance/recycling } \\
\text { requirements } \\
\text { 9. Material requirements } \\
\text { 10. Market/company } \\
\text { constraints } \\
\text { 11. Patents \& standards } \\
\text { 12. Prototype testing } \\
\text { requirements } \\
\text { 13. Schedule } \\
\text { requirements } \\
\text { 14. Prototype iteration } \\
\text { 15. Cost and time } \\
\text { requirements } \\
\text { 16. Product interactions } \\
\text { and experiences } \\
\text { 17. Government } \\
\text { regulations, } \\
\text { 18. Enabling technology, } \\
\text { etc. }\end{array}$ & $\begin{array}{l}\text { 1. Sketches with } \\
\text { annotation } \\
\text { 2. Low fidelity model } \\
\text { 3. Ideas with other } \\
\text { forms like text } \\
\text { 4. Scenario/research } \\
\text { boards } \\
\text { 5. Idea selection } \\
\text { 6. Concept feedback, } \\
\text { etc. }\end{array}$ & $\begin{array}{l}\text { 1. Sketching } \\
\text { 2. Intention map } \\
\text { 3. CAD models with } \\
\text { different levels of detail } \\
\text { and quality } \\
\text { 4. Physical models } \\
\text { 5. Rendering } \\
\text { 6. Materials } \\
\text { 7. Interacting process } \\
\text { 8. Product structure } \\
\text { 9. Mechanisms } \\
\text { 10. Costing } \\
\text { 11. Prototype iterations } \\
\text { 12. Concept prototype } \\
\text { feedback } \\
\text { 13. Evaluation criteria } \\
\text { 14. Feasibility } \\
\text { 15. Compatibility } \\
\text { 16. Completeness } \\
\text { 17. Cost evaluation } \\
\text { 18. Evaluation methods } \\
\text { 19. Selection criteria } \\
\text { 20. Stakeholders' review } \\
\text { etc. }\end{array}$ & $\begin{array}{l}\text { 1. Complete specification } \\
\text { of the geometry, material, } \\
\text { and tolerances of all parts } \\
\text { 2. Detail and assembly } \\
\text { drawings } \\
\text { 3. Parts list with raw } \\
\text { material sizes and } \\
\text { specifications } \\
\text { 4. Bill of materials (BOM) } \\
\text { 5. Selection of materials } \\
\text { 6. CAx models } \\
\text { 7. Manufacturing } \\
\text { techniques } \\
\text { 8. Machine tools and } \\
\text { processes } \\
\text { 9. Analysis results of the } \\
\text { robustness \& performance } \\
\text { of components and } \\
\text { assemblies } \\
\text { 10. Impact of design } \\
\text { decisions on the } \\
\text { performance, reliability, } \\
\text { and cost of the product } \\
\text { 11. Prototype control plan } \\
\text { 12. Prototype verification } \\
\text { tests } \\
\text { 13. Evaluation criteria } \\
\text { 14. Stakeholders' review, } \\
\text { etc. }\end{array}$ & $\begin{array}{l}\text { 1. Company's policies } \\
\text { \& procedures } \\
\text { 2.Company culture } \\
\text { 3. Compliance \& legal } \\
\text { regulations } \\
\text { 4. Tools to deliver a } \\
\text { service } \\
\text { 5. Service information } \\
\text { flow } \\
\text { 6. Interactive } \\
\text { touchpoints } \\
\text { 7. Service evaluation } \\
\text { 8. Environment } \\
\text { interactions } \\
\text { 9. Service strategy } \\
\text { 10. Service blueprints } \\
\text { 11. Experience } \\
\text { prototypes } \\
\text { 12. Users' iterations } \\
\text { 13. Service plan } \\
\text { 14. User feedback, etc. }\end{array}$ \\
\hline
\end{tabular}

Fig. 4 Key information items at product pre-design and design stages 
Table 1 Exchanged information supported by the existing product data exchange models

\begin{tabular}{|c|c|c|}
\hline Attribute & $\begin{array}{l}\text { STEP Application } \\
\text { Protocol AP 203, } \\
\text { AP214, and AP242 }\end{array}$ & $\begin{array}{l}\text { CPM and } \\
\text { its } \\
\text { extensions }\end{array}$ \\
\hline $\begin{array}{l}\text { Geometry (point, line, plane, } \\
\text { wireframe, surface models, } \\
\text { faceted models, manifold } \\
\text { surfaces and solids, } \\
\text { constructive solid geometry, } \\
\text { hybrid models, etc.) }\end{array}$ & • & • \\
\hline Function & • & • \\
\hline Form & - & - \\
\hline Behaviour & • & • \\
\hline Material & • & - \\
\hline Flow & & • \\
\hline Colours and layers & • & \\
\hline $\begin{array}{l}\text { Textual annotations associated to } \\
\text { the geometry }\end{array}$ & • & \\
\hline Data for configuration control & • & \\
\hline $\begin{array}{l}\text { Relationship (association, } \\
\text { constraint, usage, and trace } \\
\text { relationships) }\end{array}$ & & • \\
\hline Specification & & • \\
\hline Requirement & & - \\
\hline $\begin{array}{l}\text { Information (a brief textual } \\
\text { description slot, a textual } \\
\text { documentation string, a } \\
\text { properties slot that contains a } \\
\text { set of attribute-value pairs } \\
\text { stored as strings representing all } \\
\text { domain- or object-specific attri- } \\
\text { butes) }\end{array}$ & & • \\
\hline $\begin{array}{l}\text { References to product data } \\
\text { represented in another format } \\
\text { than STEP }\end{array}$ & - & \\
\hline Process information & & • \\
\hline $\begin{array}{l}\text { Data related to the documentation } \\
\text { of design change process, } \\
\text { approval, security classification }\end{array}$ & - & \\
\hline $\begin{array}{l}\text { Rationale (attributes that record } \\
\text { explanatory information on the } \\
\text { reasons for or justifications of a } \\
\text { particular decision in the } \\
\text { product development process) }\end{array}$ & & • \\
\hline Assemblies and parts & • & - \\
\hline $\begin{array}{l}\text { Relative position and orientation } \\
\text { of assembly and part }\end{array}$ & & • \\
\hline $\begin{array}{l}\text { Connection and association } \\
\text { relationship among assemblies } \\
\text { and parts }\end{array}$ & & - \\
\hline $\begin{array}{l}\text { Tolerance information including } \\
\text { dimensional tolerance and } \\
\text { geometric tolerance (form, } \\
\text { profile, runout, orientation, and } \\
\text { location tolerances) }\end{array}$ & • & $\bullet$ \\
\hline $\begin{array}{l}\text { Validation properties (global as } \\
\text { volume, area, left; local as } \\
\text { clouds of points) }\end{array}$ & • & \\
\hline Construction history in 3D & • & \\
\hline
\end{tabular}

Table 1 (continued)

\begin{tabular}{lll}
\hline Attribute & $\begin{array}{l}\text { STEP Application } \\
\text { Protocol AP 203, } \\
\text { AP214, and AP242 }\end{array}$ & $\begin{array}{l}\text { CPM and } \\
\text { its } \\
\text { extensions }\end{array}$ \\
\hline $\begin{array}{l}\text { Definitions for PDM specially } \\
\text { configuration management }\end{array}$ & & \\
$\begin{array}{l}\text { Tools used by manufacturing } \\
\text { Information for process plan, } \\
\text { configuration control }\end{array}$ & - & \\
$\begin{array}{l}\text { Parametric assembly constraints } \\
\text { Relationship among assembly } \\
\text { features }\end{array}$ & & - \\
$\begin{array}{l}\text { Kinematic pair/structures } \\
\text { Rules and constraints }\end{array}$ & • & - \\
\hline
\end{tabular}

machinery, measurement, and environment. Unlike DFMEA [37], the PFMEA mainly focuses on current PDD processes to identify potential risks and problems, enabling PDD to be performed in a reliable and efficient process with minimum manufacturing and assembly risks. The information collected through PFMEA includes the operation intent (process function), potential failure modes, list of effects of each failure, severity ranking, causes of failure, current process controls, etc. Whenever there is a change to the design process due to the failure analysis, the PFMEA should be reconducted. Correspondingly, control plans could be changed as well after PFMEA. In practice, the key information covered by a production control plan (in the form of worksheets) includes supplier information, support equipment, product and process characteristics, specification and tolerance, evaluation method, control method, and reaction plan, etc. In the manufacturing process, control plans and PFMEA could help manufacturers diagnose their quality challenges in product design, design for manufacturing solutions, and suggestion to design changes. Since the proposed PDLIM accommodates the key information items covering PFMEA and control plan worksheets as information values, so the PDLIM could be easily integrated into the DFMEA and PFMEA in practice.

Like in S3, the key information items for stages S4 and S5 are extracted and listed in Fig. 6. The information collected in these two phases is usually treated as the starting point of or driving factors for the next round product design process.

The design information at product pre-design, design, and manufacturing stages has great influence on the current generation product quality, while that at stages S4 and S5 has more impacts on next generation product design. The manufacturers have limited or no control on products when they are sold out, leading to the limited feedback from these stages such as product real-time usage data, product strong points and weak points, user interaction behaviour data, product operational environment, product real-time performance, maintenance history, etc. Whereas this feedback is useful in 


\begin{tabular}{|c|c|c|c|c|c|}
\hline $\begin{array}{l}\text { Design } \\
\text { Phase }\end{array}$ & $\begin{array}{c}\text { Manufacturing } \\
\text { scheduling (S3-P1) }\end{array}$ & $\begin{array}{c}\text { Actual parts } \\
\text { manufacturing(S3-P2) }\end{array}$ & $\begin{array}{l}\text { Product assembly } \\
\text { (S3-P3) }\end{array}$ & $\begin{array}{l}\text { Product testing } \\
\text { (S3-P4) }\end{array}$ & $\begin{array}{c}\text { Product } \\
\text { transportation(S3-P5) }\end{array}$ \\
\hline $\begin{array}{c}\text { Key } \\
\text { information } \\
\text { items }\end{array}$ & $\begin{array}{l}\text { 1. Raw materials } \\
\text { availability in stock } \\
\text { 2. Cost of raw materials } \\
\text { 3. Material \& component } \\
\text { orders } \\
\text { 4. Suppliers' lead times } \\
\text { and prices } \\
\text { 5. Availability of } \\
\text { workstations and workers } \\
\text { 6. Information about other } \\
\text { works on the machine } \\
\text { 7. Manufacturing capacity } \\
\text { and productivity } \\
\text { 8. Costs related to } \\
\text { machines and workers on } \\
\text { the workstations } \\
\text { 9. Scheduling tool } \\
\text { 10. Schedule of } \\
\text { collaborators } \\
\text { 11. Plan for conflicts } \\
\text { 12. Implementation plan, } \\
\text { 13. Control plan, etc. }\end{array}$ & $\begin{array}{l}\text { 1. Monitoring of } \\
\text { manufacturing process } \\
\text { 2. Manufacturing } \\
\text { environment } \\
\text { 3. Government } \\
\text { manufacturing } \\
\text { regulations } \\
\text { 4. Changes to } \\
\text { components/parts } \\
\text { 5. Changes to } \\
\text { manufacturing process } \\
\text { 6. Changes to } \\
\text { manufacturing } \\
\text { technology } \\
\text { 7. Performance indicators } \\
\text { 8. Manufacturing flow } \\
\text { 9. Actual material cost } \\
\text { 10. Actual labour cost } \\
\text { 11. Actual overhead cost } \\
\text { 12. Interaction with } \\
\text { collaborators } \\
\text { 13. Checking quality and } \\
\text { accuracy of parts against } \\
\text { the design specifications } \\
\text { 14. Stakeholders' review, } \\
\text { etc. }\end{array}$ & $\begin{array}{l}\text { 1. Assembly cost } \\
\text { 2. Assembly standards } \\
\text { 3. Assembly process } \\
\text { 4. Functional requirements } \\
\text { 5. Functional analysis } \\
\text { 6. considerations/ } \\
\text { Assumptions } \\
\text { 7. Number of parts } \\
\text { 8. Number of interfaces } \\
\text { 9. Mistake proofing } \\
\text { 10. Handling } \\
\text { 11. Insertion } \\
\text { 12. Secondary operations } \\
\text { 13. Checking fit and } \\
\text { function against parts } \\
\text { accuracy/drawings/design } \\
\text { intent, etc. }\end{array}$ & $\begin{array}{l}\text { 1. Testing standard and } \\
\text { criteria } \\
\text { 2. Product usability } \\
\text { 3. Product safety } \\
\text { 4. Product function test } \\
\text { 5. Product reliability } \\
\text { 6. Testing methods } \\
\text { 7. Testing technology } \\
\text { 8. Work experience and } \\
\text { professional levels of } \\
\text { testing personnel } \\
\text { 9. Testing } \\
\text { manufacturing } \\
\text { procedure } \\
\text { 10. Design defects } \\
\text { 11. Manufacturing } \\
\text { defects } \\
\text { 12. Testing product } \\
\text { lifespan } \\
\text { 13. Potential failure } \\
\text { mode, effects, causes } \\
\text { 14. Recommend actions } \\
\text { 15. Product competition, } \\
\text { etc. }\end{array}$ & $\begin{array}{l}\text { 1. Packaging } \\
\text { 2. Transportation } \\
\text { mode selection } \\
\text { 3. Transportation cost } \\
\text { 4. Transportation } \\
\text { strategy } \\
\text { 5. Customer } \\
\text { requirements } \\
\text { 6. Carrier relationships } \\
\text { 7. carrier's } \\
\text { performance } \\
\text { 8. Product } \\
\text { transportation track } \\
\text { history } \\
\text { 9. Transportation } \\
\text { quality } \\
\text { 10. Transportation } \\
\text { efficiency } \\
\text { 12. Sustainability } \\
\text { carbon footprint } \\
\text { 13. Time cost, etc. }\end{array}$ \\
\hline
\end{tabular}

Fig. 5 Key information items at product manufacturing stage

\begin{tabular}{|c|c|c|c|c|}
\hline $\begin{array}{l}\text { Design } \\
\text { Phase }\end{array}$ & $\begin{array}{l}\text { Product launch } \\
\text { (S4-P1) }\end{array}$ & $\begin{array}{l}\text { Product in use } \\
\text { (S4-P2) }\end{array}$ & $\begin{array}{c}\text { Product maintenance } \\
\text { (S5-P1) }\end{array}$ & $\begin{array}{c}\text { Product recycling } \\
\text { (S5-P2) }\end{array}$ \\
\hline $\begin{array}{c}\text { Key } \\
\text { information } \\
\text { items }\end{array}$ & $\begin{array}{l}\text { 1. Launch date } \\
\text { 2. Product promotion } \\
\text { channels } \\
\text { 3. Sales strategy and } \\
\text { training } \\
\text { 4. Technical data sheets } \\
5 \text {. Technical support } \\
\text { materials } \\
\text { 6. Product launch cost } \\
\text { 7. Infrastructure } \\
\text { changes } \\
8 . \text { Communications plan } \\
\text { both inside and outside } \\
\text { the organization } \\
9 . \text { Analyst briefings } \\
\text { 10. Product propagate } \\
\text { 11. Technical data } \\
\text { sheets } \\
\text { 12. Brand development, } \\
\text { etc. }\end{array}$ & $\begin{array}{l}\text { 1. Product real-time } \\
\text { conditions } \\
\text { 2.Product operational } \\
\text { environment } \\
\text { 3. Feedback from end } \\
\text { users } \\
\text { 4. User behavior data } \\
\text { 5. Tools for collecting } \\
\text { product-generated and } \\
\text { user-generated data } \\
\text { 6. real-time data } \\
\text { analysis tool } \\
\text { 7. Real life } \\
\text { observational study } \\
\text { 8. Forecasting future } \\
\text { service, etc. }\end{array}$ & $\begin{array}{l}\text { 1. Failure data and } \\
\text { causes } \\
\text { 2. Product } \\
\text { maintenance record } \\
\text { 3. component/part } \\
\text { status and quality } \\
\text { 4. Maintenance } \\
\text { service providers } \\
\text { 5. Maintenance cost } \\
\text { 6.Condition } \\
\text { evaluation } \\
\text { 7. Fault diagnosis } \\
\text { 8. Maintenance } \\
\text { service quality } \\
\text { 9. Maintenance time } \\
\text { 10. Cost measures, } \\
\text { etc. }\end{array}$ & $\begin{array}{l}\text { 1.The cost of } \\
\text { recycling and } \\
\text { disassembly } \\
\text { 2. The reusable state } \\
\text { 3. Remaining service } \\
\text { time of parts/ } \\
\text { components } \\
\text { 4. Recycling } \\
\text { technology } \\
\text { 5. Recycling process } \\
\text { 6. Revenue from } \\
\text { product recycling } \\
\text { 7. Material recycling } \\
\text { 8. Recycling service } \\
\text { 9. Percentage of } \\
\text { recycled products, etc. }\end{array}$ \\
\hline
\end{tabular}

Fig. 6 Key information items at product launch \& in use and maintenance \& recycling stages 
helping manufacturers respond to the upcoming maintenance in advance and guiding product design and manufacturing in the next round [4].

\subsection{Design environment associated information}

The PDD process usually involves various participants within different teams working collaboratively to process various information to reach a detailed design, along which design information evolves over time. Comparing to many other processes, the PDD process is especially challenging to manage as it tends to involve significant elements of novelty, complexity, and iteration [40]. In a PDD process, design tasks can be performed by in-house design teams in a traditional design environment or by both internal staff and external participants in a combined design environment with crowdsourcing. The key information items related to a traditional or combined crowdsourcing design environment can be explored from a design quality control and assurance point of view [7].

\subsubsection{Associated information in traditional in-house design environment}

In a traditional in-house design environment, the associated information mainly comes from the following three aspects:

(1) Product family or lifecycle changes-related information, which is typically managed by the PDM [27] tool or integrated PLM tool $[28,35]$.

(2) Design team, IP and supply-chain related information, which is typically managed by the ERP system in manufacturing stages.

(3) Product and end-user generated information, which could be managed by DT platform.

The existing PDM/PLM and ERP systems form the basis for improving product quality, but there still lacks product real-time performance information and user interaction information for better data-informed product design. Currently, the most suitable technology to obtain and use such data information along the product lifecycle is DT enabled by IoT and sensors. DT has been listed as one of the top 10 technology trends in 2018 [41]. It provides a means of connecting information such as real-time status of the physical product and its behaviour in the physical world with a digital representation, enabling companies or users to have a real-time view of the product and its usage in the physical space thus to adopt evidence-based actions on physical asset management [42]. Meanwhile, it provides a channel for customers to provide their feedback, offering value in operational efficiency and insights into how products are used and how they can be improved in the next round of product development process. Tao et al. [16] have put forward a DT-driven design framework to ensure that useful customer voices from online customer reviews would be considered for decision makings for redesigning the existing products.

So far, DT is mainly applied at product manufacturing and use stages, where most of the near real-time data such as product realtime conditions, operating environments, and non-real-time data like customers' comments/feedback on products come from. Benefited from the cheap and miniaturized sensors that can be embedded into products, all possible kinds of imaginable data including text, audio, RGB images and videos can be transmitted to a specific digital-twin server with high sampling frequency, offering a real-time look at what's happing with physical assets. The information acquired by sensors could not only help reduce maintenance costs and pains drastically, but also drive the improvement of current products [10]. For obtaining customers' feedback, online surveys and activities on social networking sites and crowdsourcing are adopted. The acquired data are analyzed by data scientists (or agents) for uncovering new business opportunities and optimizing product and process designs by continuous evaluation.

Although the ability of capturing the real-time data is enabled by IoT, it is challenging to store and process them in real-time [43]. Therefore, the combination of Redis and MongoDB database could be helpful. Redis acts as cache database for quick data processing while MongoDB is used for long-term data storage. The data in MongoDB is migrated from Redis at a certain frequency. Before the collected data are analyzed, data pre and post processing like data standardization, the filtering of duplicate data, and data fusion with other structured and unstructured data should be performed to ensure the analysis performance.

For successful implementation of DTs, blockchain is believed to play an important role in data security and trust due to its inherent decentralized nature [43]. It stores information in blocks that are verified by peer-to-peer networks before they are linked to the blockchain using a cryptographic hash generated from the contents of the previous block. It ensures that the generated and operational data in the product lifecycle actually originate from trustworthy sources and spread across among trusted parties, enabling maximum security, traceability and transparency in applications. Furthermore, the implementation of DTs on blockchain can eliminate fake products from the market [10]. There are already some studies focusing on how to integrate DT with blockchain to ensure data security and reliability. For example, Reyna et al. [44] investigates the challenges of integrating blockchain with IoT that is one of the key enablers to DT in terms of storage capacity and scalability, anonymity and data privacy, and security, etc.

As this research is mainly focused on what information is necessary for supporting the product design trends in combined design environments, we only provide a possible solution on how to process the real-time DT data in terms of data acquisition, storage, security, and necessary pre and post data processing. 
Thus, the information managed by PDM/PLM and ERP systems and the DT platform is also covered by the PDLIM as part of the traditional environment information (see Fig. 7).

In Fig. 7, some key information items collected by DT platforms are the same with those in the product use phase (S4-P2), but the information volumes and accuracies to the corresponding items are quite different. In S4-P2, the information is mainly collected by traditional ways such as customer reviews, questionnaire survey, and focus groups, while the DT information is collected by product embedded IoT sensors and from the Internet. The data volume from DT is much larger than that in S4-P2, and the data accuracy is much finer.

\subsubsection{Associated information in crowdsourcing design environment}

To shorten the lead time to market, SMEs are increasingly adopting concurrent design strategies to get core design tasks conducted by in-house design teams/departments while crowdsourcing less important ones to the crowds. Although diverse crowdsourcing models have been implemented by existing crowdsourcing systems, they have the same key components affecting product design quality, namely, the requester/crowdsourcer, the crowds (workers), the task, and the platform $[45,46]$. When a design task at the early design stage is crowdsourced, the crowds could be further classified into the designer and the evaluator. According to the crowdsourcing quality model [8], the output (data/product) quality is determined by people quality, process quality and software/ crowdsourcing platform quality. Therefore, the information identified in crowdsourcing design environments is mainly extracted into the following six categories [7, 8] (see Fig. 8).

\subsection{PDLIM application scenarios and potential benefits}

In product design practice, SMEs usually adopt the hybrid innovation strategy to learn from the past and their competitors including previous product generations and user reviews/ comments on existing products. In our previous research, Zhang et al. [1] have proposed a feature-based product family design framework for developing a new generational product design based on the previous generational product designs and user experience information. Cheng et al. [47] have developed a method for interpreting user experience from reviewing user comments, which can be used for the identification of user needs for a new product development. But, to support this hybrid innovation strategy, there is a need for a product design lifecycle information model to support related information capturing and reusing, which can support the hybrid innovation strategy in incremental product family development. How the proposed PDLIM can be used to support the hybrid innovation strategy in product family design is illustrated in Fig. 9, which shows the key information relationships between two product generations. The main forward information flows between product lifecycle development stages are represented by blue arrows. Other four design information flows represented by red, green, black, and yellow line arrows are illustrating four typical application scenarios (relationships) of the PDLIM.

Firstly, at the beginning (S1) of the PDD process of the first generation product, there is always much design information needed for competitor products analysis and identification of market and user needs. This information is then used to develop and evaluate the new product design specification. Afterwards, the product is designed at S2, manufactured at $\mathrm{S} 3$, used at S4, maintained and recycled at S5. Within this product generation, a specific product design phase is mainly

\begin{tabular}{|c|c|c|c|}
\hline $\begin{array}{c}\text { In-house } \\
\text { design } \\
\text { environment }\end{array}$ & $\begin{array}{l}\text { Product information managed } \\
\text { by PDM/PLM (E1-F1) }\end{array}$ & $\begin{array}{l}\text { Manufacturing information } \\
\text { managed by ERP (E1-F2) }\end{array}$ & $\begin{array}{c}\text { Product and end user generated } \\
\text { information managed by DT } \\
\text { platforms (E1-F3) }\end{array}$ \\
\hline $\begin{array}{l}\text { Key } \\
\text { information } \\
\text { items }\end{array}$ & $\begin{array}{l}\text { 1. Real-time BOM information } \\
\text { 2. Details for understanding and } \\
\text { approving engineering change requests } \\
\text { 3. Engineering change orders } \\
\text { 4. real-time product development } \\
\text { status } \\
\text { 5. Milestone tracking during } \\
\text { development process } \\
\text { 6. Data version control } \\
\text { 7. Visualization of involved } \\
\text { stakeholders } \\
\text { 8. Approved manufacturer lists } \\
\text { 9.Property parameters } \\
\text { 10. Management team, etc. }\end{array}$ & $\begin{array}{l}\text { 1. Team structure } \\
\text { 2. Team members' skills } \\
\text { 3. Company culture, rules \& policies } \\
\text { 4. risks } \\
\text { 5. Supply chain operations } \\
\text { 6. Team members' satisfaction } \\
\text { 7.Team members' education } \\
\text { background } \\
\text { 8. Record of purchasing } \\
\text { 9. Inventory and procurement } \\
\text { 10. Process parameters, etc. }\end{array}$ & $\begin{array}{l}\text { 1. Real-time product conditions } \\
\text { 2. Product's operating environment } \\
\text { 3. Customers' comments } \\
\text { 4. Historical product operation data } \\
\text { 5. Historical interaction data between } \\
\text { physical and virtual twins, } \\
\text { 6. Connection to data sources, etc. }\end{array}$ \\
\hline
\end{tabular}

Fig. 7 Key information items related to traditional in-house design environment 


\begin{tabular}{|c|c|c|c|c|c|c|}
\hline $\begin{array}{l}\text { Crowdsourcing } \\
\text { environment }\end{array}$ & $\begin{array}{l}\text { Crowds/service provider } \\
\text { (E2-F1) }\end{array}$ & Task (E2-F2) & Requester (E2-F3) & Platform (E2-F4) & $\begin{array}{l}\text { Crowdsourcing } \\
\text { process (E2-F5) }\end{array}$ & $\begin{array}{c}\text { Information } \\
\text { management (E2-F2) }\end{array}$ \\
\hline $\begin{array}{c}\text { Key } \\
\text { information } \\
\text { items }\end{array}$ & $\begin{array}{l}\text { 1. Work histories } \\
\text { 2. Location } \\
\text { 3. Qualification } \\
\text { 4. Reputation/history } \\
\text { comments } \\
\text { 5. Expertise } \\
\text { 6. Participation motivation } \\
\text { 7. Time or schedule of } \\
\text { crowds, etc. }\end{array}$ & $\begin{array}{l}\text { 1. Task definition } \\
\text { 2. Task decomposition } \\
\text { 3. Technical resources } \\
\text { 4. Incentives mechanism } \\
\text { 5. Task input and output } \\
\text { 6. Task distribution } \\
\text { process } \\
\text { 7. Timeline, etc. }\end{array}$ & $\begin{array}{l}\text { 1. Open call design } \\
\text { 2. Privacy provision } \\
\text { repositories } \\
\text { 3. Ethicality provision } \\
\text { 4. Incentives provision, } \\
\text { etc. }\end{array}$ & $\begin{array}{l}\text { 1. Platform performance } \\
\text { 2. Interaction tools for } \\
\text { crowds and requester } \\
\text { 3. Platform facilities } \\
\text { 4. Platform pattern and } \\
\text { characteristics, etc. }\end{array}$ & $\begin{array}{l}\text { 1. Crowds selection } \\
\text { criteria } \\
\text { 2. Open task design } \\
\text { requirements } \\
\text { 3. Interaction among } \\
\text { crowds } \\
\text { 4. Design team } \\
\text { establishment, etc. }\end{array}$ & $\begin{array}{l}\text { 1. Information structure } \\
\text { 2. Information repositories } \\
\text { 3. The performance of } \\
\text { information management } \\
\text { software } \\
\text { 4. Service information } \\
\text { management } \\
\text { 5. Information reliability, } \\
\text { etc. }\end{array}$ \\
\hline
\end{tabular}

Fig. 8 Key information items related to crowdsourcing design environment

affected by the information collected at previous design phases (forward information flows), but the integrated service design phase (S2-P4) can also be affected by the information collected at product launch, in use, maintenance, and recycling phases (information relationship 1). Although the product has been designed, manufactured and in use, new integrated services at S2-P4 for the current generation product can still be designed and developed incrementally such as new repairing/maintenance/leasing services. When designing such new services, the information gathered in S4 and S5 can feed backward to new integrated service design at S2-P4 for bettering total user experience and business values. This information relationship is shown in red line arrows.
Secondly, if the first generation product needs to be redesigned/ upgraded, it enters to the next (second) generation product design in a product family. The product design lifecycle information related to the first generation product would affect the second generation product design. For example, the information gathered in S4 and S5 of the first generation product will feed forward to the $\mathrm{S} 1$ of the second generation product design (illustrated in green line arrows - information relationship 2) to overcome the product drawbacks identified in the previous generation. While the information gathered at $\mathrm{S} 2$ and $\mathrm{S} 3$ of the first generation product will correspondingly affect $\mathrm{S} 2$ and $\mathrm{S} 3$ in the second generation product development (illustrated in black line arrows - information relationship 3), where the corresponding design information at the first

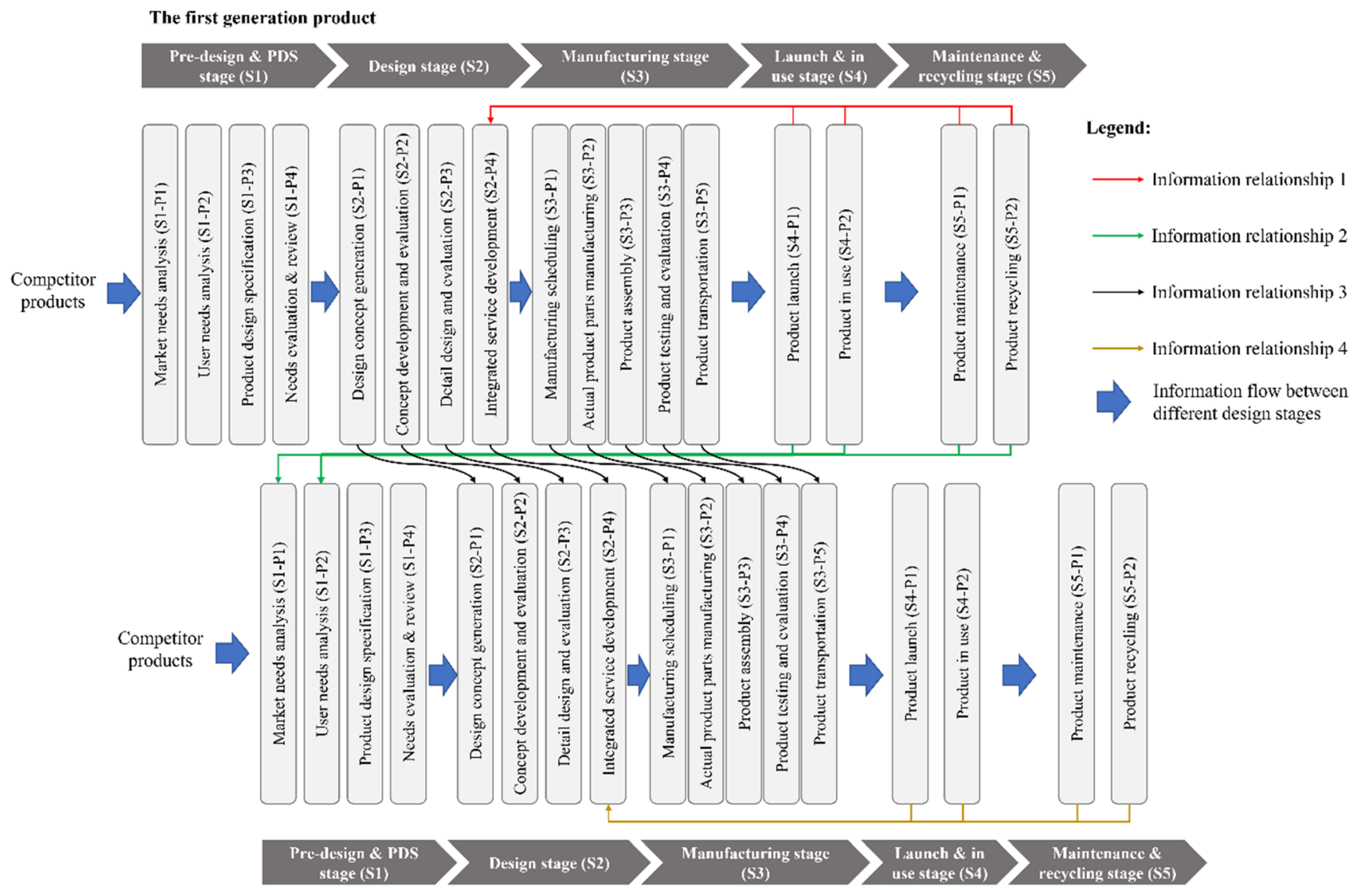

The second product generation

Fig. 9 Information relationships between two generation products 
generation product can be treated as the basis for further product family design and manufacturing.

Finally, after the second generation product is launched on the market, the information collected at its S4 and S5 can be further used at its previous phase S2-P4 for new integrated service design and development (illustrated in yellow line arrows - information relationship 4). This is similar to information relationship 1.

With the four information relationships identified within two generation products, the design decision-making could be better informed based on the available holistic information along the product design lifecycle at each design phase. Considering product design lifecycle information in the PDLIM has the following potential benefits:

Greatly supporting new integrated service design for the current generation product and next generation new product designs as we explained above.

Improved decision-making quality in the product design and development process Decision-making is a time-critical task that requires the support of useful design information. In design practice, due to the large volume of design information, it is hard for decision-makers to get the right information for the right purpose at the right time [48]. The PDLIM wardrobe structure can provide a big picture about the structured design information throughout the product design lifecycle, enabling easy access to useful design information. If an information management system for design information storage, retrieval, processing, and analysis is implemented, then the decision-making process would be improved.

Increased enrichment of design information/data With the PDLIM, SMEs could incrementally enrich their product and associated services design over time, forming the basis for future data-driven product design and development supported by big data analytics and artificial intelligence.

Increased product design richness The proposed PDLIM can help accelerate product design process by organizing available design information in a holistic and structural way, especially useful in PFD.

\section{Qualitative evaluation of identified information items in the PDLIM}

The proposed PDLIM has been evaluated in two steps. First, the necessity (or usefulness) of the primary information items in the three main information layers are qualitatively evaluated via two case studies. Second, the secondary interaction information on the "Interaction" layer is qualitatively evaluated with examples, which are detailed in next section.

The first case study is from a training program for Industrial Design undergraduate at Northumbria School of
Design, illustrating the primary information items in product pre-design and design stages. The design briefs, Critical Justification/design notebook and presentation documents are analyzed to verify the identified design information by various information representation/value examples. The second one is from existing literatures mainly focusing on the primary design information in a product family design of sharing bicycles from docked generation to dockless generation.

\subsection{Case study 1—“Redesigning the food processor"}

This case study is set up by a kitchen appliance manufacturer that expects to shift its product design trends from baking to food preparation for meals that are healthier and fit into a balanced diet. The given design brief provides the information about the brand vision, the product history, the segmentation of target customers, company design language, and company insights, etc. The design information in the design brief constraints the design of new food processor products. For example, the new food processor must be designed with the company design language and in line with the current kitchen appliance style. From this point of view, the design of the current food processor product is affected by previous generation products. Since the case is an undergraduate training project, so it mainly concerns related design information at both product pre-design and design stages where forward information flows are mainly emphasized.

To achieve the design aim, design activities such as personas and reasoning, design research, market research, concept development, and storyboarding have been conducted. Based on the product design presentation document, the key information items and the corresponding information values (data entities) at each design phase are extracted and shown in Table 2. In this case study, any design phase listed in Table 2 can be conducted in either a traditional design environment or a crowdsourcing combined environment.

In this case study, although geometry information of the food processor design can be easily traced through CAD systems/tools, there is still much information in pre-design and design stages that cannot be processed by CAD tools and is usually recorded in the design notebooks and portfolios with various formats, such as hand-drawn sketches, images, texts, tables, and physical prototypes with various materials. The proposed PDLIM likes an information wardrobe with a structure (see the first column in the Table 2) and many drawers. Each drawer can have a label to describe a key design information item at a higher level of abstraction (see the second column in Table 2) and an inner space for storing a varied number of detailed information and data entities' (or information values') locations. As a large volume of design information in various formats (see the third column in the Table 2) such as 3D models, drawings, sketches, graphs, and worksheets would be created 
Table 2 Design phases, key information items, and information values generated from case study 1

\begin{tabular}{ll} 
Design phase & Key information items and their codes \\
\hline $\begin{array}{l}\text { Market needs analysis } \\
\text { (S1-P1) }\end{array}$ & Development/market trends (S1-P1-2)
\end{tabular}

Innovation strategy (S1-P1-12)

Brand audit (S1-P1-13)

Competitive advantage (S1-P1-14)

User needs analysis (S1- Customer personas (S1-P2-3)

P2)

Product design specification (S1-P3)

Design concept generation (S2-P1)

Concept development $\&$ evaluation (S2-P2)

Detail design and evaluation (S2-P3)
Frustrations/pain points for each customer segment (S1-P2-4)

Material requirements (S1-P3-9)

Market/company constraints (S1-P3-10), constrained by S1-P1-12 and S1-P1-13

Sketches with annotation (S2-P1-1)

CAD models with different levels of detail and quality (S2-P2-3)

Colours (S2-P2-5)

Detail and assembly drawings (S2-P3-2)

Selection of materials (S2-P3-5)

CAx models (S2-P3-6)

\section{Information representation/value example}
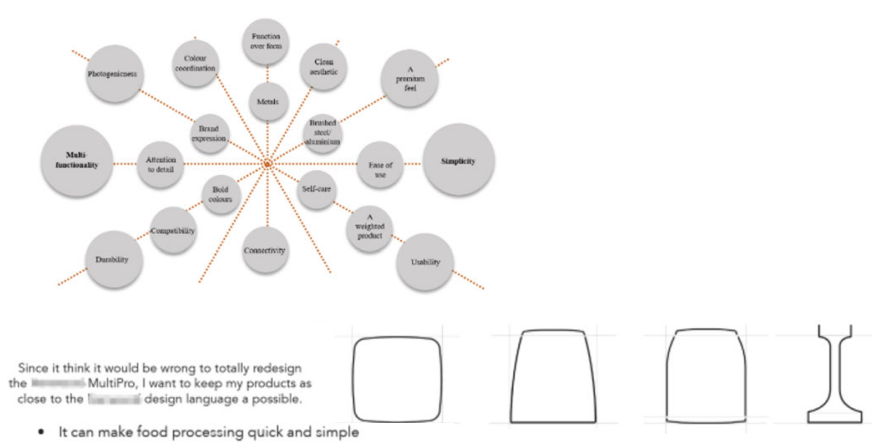

- It is multifunctional

- It can be used for a plethora of dishes and food preparation.
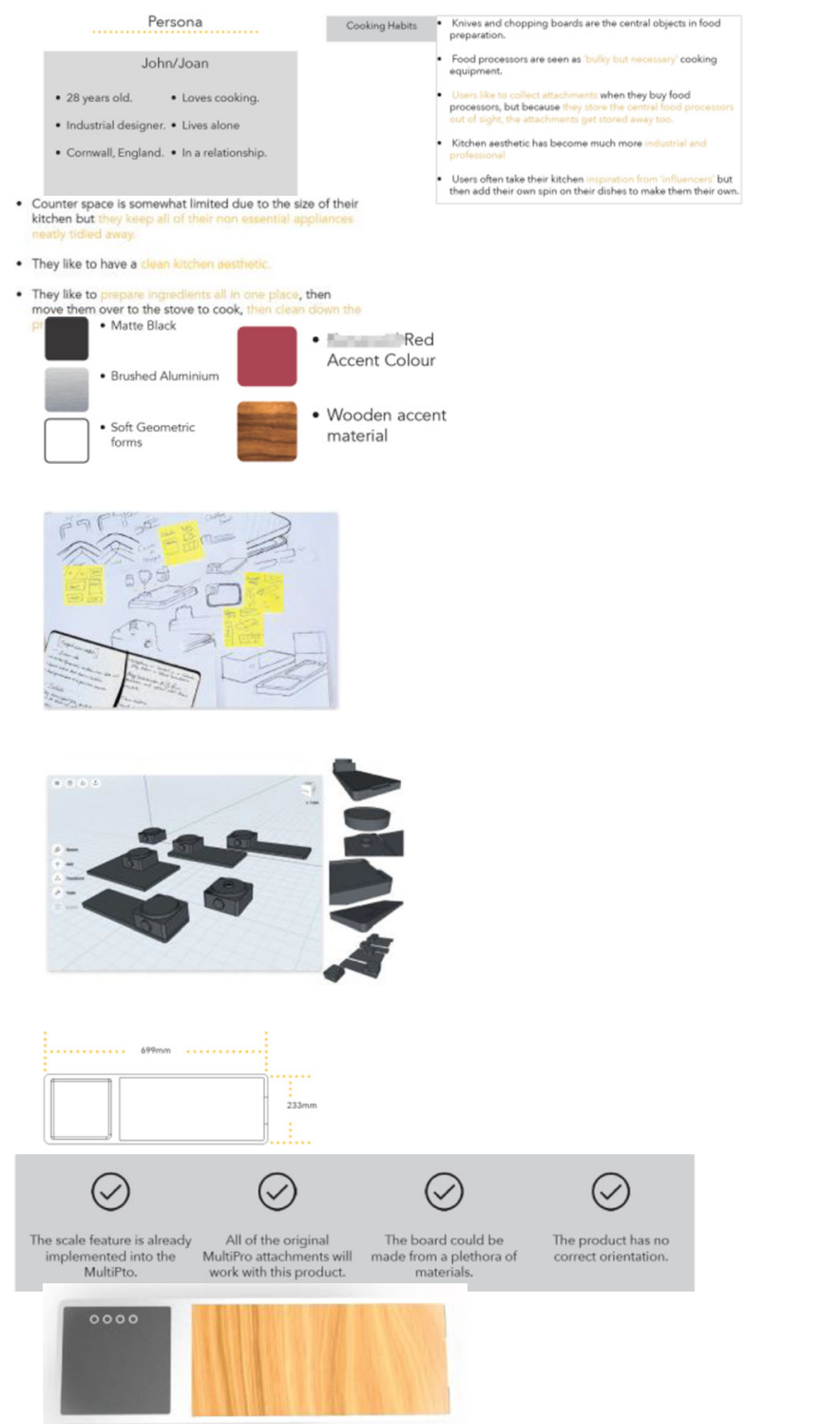
Table 2 (continued)

\begin{tabular}{lll}
\hline Design phase & Key information items and their codes & Information representation/value example \\
\hline Product verification tests (S2-P3-11)
\end{tabular}

in the food processor design process, it is impossible to store all of them in a database, so a MongoDB database is adopted to manage these data or their storage location so that the decision-makers can access this information when needed. An example of how to model key design information items is illustrated in Fig. 12 in Section 5.2. Note that comparing with all identified key information items in the PDLIM shown in Fig. 4, not all of them are listed in the second column of Table 2 for this specific product design case, that is just like a wardrobe, not all drawers are used all the time. Secondly, for each key information item, specified information and data entities to describe it are varying in terms of the volume of information and data formats. But the PDLIM does provide a design information wardrobe structure containing all designed drawers to provide structured spaces for storing the classified key information items in a product lifecycle. Some raw design information entities such as $3 \mathrm{D}$ physical prototypes could be pre-processed for easy-storing and communication such as 3D scanning of 3D physical prototypes into 3D models. This case study not only shows the relationship between the identified information items in the PDLIM and design case-based information values (or data entities), but also illustrates how to use the PDLIM in practice. As for how to model and process each data entity such as pictorial data in this case, the data can be created from various design tools such as Photoshop and CAD packages. After that, the data entity can be shared via various digital data sharing tools such as Microsoft OneDrive, emails, and other digital platforms and visualized in many related visualization packages. For understanding or interpreting the data entity, it can be reviewed by designers with required knowledge and experience or by AI-based digital summarization tools in future.

This case study mainly analyzed the necessity of design information at pre-design and design stages, while the second case study is conducted to verify the design information at other design stages and how the feedback from other design stages or previous product generations affects the current generation product design.

\subsection{Case study 2-“The design of dockless sharing bikes"}

This case study is to mimic product family design and product lifecycle design by taking the development of dockless sharing bikes in two generations as an example. From mid 1960s, there have been in total four generations of bike-sharing systems namely free bikes, coin-deposit system, docked IT-based system, and dockless IT-based system [49]. The first two bikesharing generations are free of charge and users are anonymous for using their services, while for later generations, they do require the users with verifiable personal ID to use their payable services.

To analyze key design information items and information values in a product family design, two different dockless Mobike $₫$ models that have been launched to the market in China were chosen. The main difference between these two Mobike ${ }^{\circ}$ models is the driving principles that lead to the difference in bike body/frame structure design. The one Mobike $₫$ model has no chain and is driven by bevel gears, while the other is driven by chain. As in the same product family, the two bike designs share the same bike components, such as basket, seat, and smart locks, etc. Key design information items and information values in these two different models at detail design and evaluation phase are shown in phase S2-P3 in Table 4.

To analyze how the design information in the previous generational design affects the current generational design as in an iterative product lifecycle design process, the development of docked and dockless IT-based bicycles is taken as an example. In the docked IT-based bicycle generation, the information identified at bicycle in use and maintenance stages is shown in Table 3. In practice, the information in Table 3 is treated as design feedback from the previous generation products and triggers the starting point for designing next generation dockless sharing bikes. With the design information identified in [49-53], the key information involved in the dockless IT-based bicycles design is extracted into Table 4. Between docked and dockless generation bicycles, information relationship 2 and 3 identified in Fig. 9 is mainly involved. For example, the user experience in bike pickups 
Table 3 Design phases, the key design information items, and information values generated from the docked bicycles

\begin{tabular}{|c|c|c|}
\hline Design phase & $\begin{array}{l}\text { Key information items and } \\
\text { their codes }\end{array}$ & Information values/representation examples \\
\hline \multirow[t]{3}{*}{$\begin{array}{l}\text { Product in use } \\
\quad(\mathrm{S} 2-\mathrm{P} 2)\end{array}$} & $\begin{array}{l}\text { Product operational } \\
\text { environment (S3-P2-2) }\end{array}$ & $\begin{array}{l}\text {-Built environment characteristics such as population density, job density, bicycle and public transit } \\
\text { infrastructure, street design, land-use mix and proximity to central areas affect the usage of } \\
\text { docked bicycles. } \\
\text {-Traffic congestion }\end{array}$ \\
\hline & $\begin{array}{l}\text { Feedback from end users } \\
\text { (S4-P2-3) }\end{array}$ & $\begin{array}{l}\text {-More organized pickup and drop-off experience for users } \\
\text {-Limited bicycles and docking spots around public transit stations influencing bicycle pickups } \\
\text { when needed and bicycle returns }\end{array}$ \\
\hline & User behavior data (S4-P2-4) & $\begin{array}{l}\text {-A substitute for walking or public transit trips } \\
\text {-The duration of trips generally falls between } 16 \text { and } 22 \text { mins } \\
\text {-Males and younger populations account for a larger percentage of docked bike-sharing users. }\end{array}$ \\
\hline \multirow[t]{2}{*}{$\begin{array}{l}\text { Product } \\
\text { maintenance } \\
\text { (S5-P1) }\end{array}$} & Maintenance cost (S5-P1-5) & $\begin{array}{l}\text {-Substantial investment required } \\
\text {-Increased maintenance costs in terms of economic and human resources with higher density of } \\
\text { docking stations }\end{array}$ \\
\hline & Maintenance time (S5-P1-9) & $\begin{array}{l}\text {-Limited distribution of stations around public transit stations } \\
\text {-Bicycles' redistribution required } \\
\text {-Drop-off restriction }\end{array}$ \\
\hline
\end{tabular}

and drop-offs with the previous generational bikes is influenced by the limited docking spots and bicycles around public transit stations (S4-P2-3 in Table 3), so the new generation dockless bicycles are designed to use smart locks (S3-P2-4 in Table 4) to remove the dock station need and make it possible for flexible bike pickups and drop-offs (information relationship 2). To accelerate dockless bicycle design and manufacturing process, the corresponding information at specific design phases such as the design of key bicycle parts of the docked generation bicycle would be helpful (information relationship 3) if both the docked and dockless bicycles are provided by the same company. As the docked bicycles have been totally replaced by the dockless ones, we mainly focus on illustrating the information relationship 4 within the dockless generation bicycle. At its use phase of dockless bicycle, it is reflected by end users that a deposit is required before use, which not only increases the users' cost but also makes it less friendly to short-term city residents. To address this problem, Mobike $®$ cancels the deposit policy and proposes more flexible service plans with different charging modes and starting prices.

In the bicycle design and development process, DFMEA and PFMEA are conducted regularly at the bicycle design and manufacturing stages to assess possible risks, thus to enable the bicycle quality and performance. As a technical system, a bicycle consists of nine subsystems, namely frame, front wheel, rear wheel, sprocket-pedal, chain-derailleur, seat, handlebar, hand brake, and suspension subsystem [54], which is presented as product/bicycle structure (S2-P2-8) in Table 4. In this case study, DFMEA can be adopted at a system, subsystem, and a component level where material properties, geometry, regulatory requirements, tolerances, interactions, and how the item behaves in its environment are all things that need to be considered. PFMEA often assumes the design is sound and its scope includes manufacturing and assembly operations, shipping, tool maintenance, etc. [54]. Examples of hand brake function for DFMEA and spoke assembly in a bicycle wheel for PFMEA are shown in information item S2-P3-9 in Table 4. In wheel assembly in PFMEA, too few spokes would lead to wheel wobbliness and increase stress during manoeuvres, with the potential for wheel collapse and rider injury. The severity scale of this serious effect is set to be 9 as it potentially affects safe operation or regulatory requirements. To address this problem, both prevention and detection design controls are currently adopted to decrease its occurrence. In addition, extra actions like the development and implementation of an in-station vision system to detect missing wheel spokes are also recommended. With DFMEA and PFMEA, the bicycle design will be reviewed regularly by a cross-functional team of engineers and members in different departments to eliminate blind spots and potential failures as much as possible. Although the DFMEA and PFMEA would iterate for several times in the bicycle design and development process, their analysis outcomes can be accommodated by the proposed PDLIM.

In summary, most products around us such as automobiles, toasters, bicycles, and vacuum cleaners are required to be iteratively designed to better meet continuously changing user needs, thus, to bring better user experience. Among their designs, bicycle design is a relatively simple example to analyze 
Table 4 Design phases, the key information items, and information values for designing the dockless bicycles

\section{Design phase Key information items and their Information values/representation examples} codes

\begin{tabular}{|c|c|c|}
\hline \multirow{3}{*}{$\begin{array}{l}\text { Market needs } \\
\text { analysis (S1-P1) }\end{array}$} & Competitor products (S1-P1-1) & OFO, Lime \\
\hline & Marketing strategies (S1-P1-3) & Targeted to specific areas or socio-demographical groups. \\
\hline & Marketing context (S1-P1-9) & $\begin{array}{l}\text { The existing large number of shared bikes has not taken into account whether these } \\
\text { systems promote the equity of bike-sharing access to all potential users, including } \\
\text { disadvantaged groups with limitations in approaching other transport modes. }\end{array}$ \\
\hline $\begin{array}{l}\text { User needs } \\
\quad \text { analysis (S1-P2) }\end{array}$ & $\begin{array}{l}\text { Frustrations/pain points for each } \\
\text { customer segment (S1-P2-4) }\end{array}$ & $\begin{array}{l}\text { The seat too low and unable to adjust; the seat cushion too stiff; no basket; most } \\
\text { importantly hard to operate due to the weight }\end{array}$ \\
\hline \multirow[t]{2}{*}{$\begin{array}{l}\text { Product design } \\
\text { specification } \\
\text { (S1-P3) }\end{array}$} & Government regulations (S1-P3-17) & $\begin{array}{l}\text { Green lifestyle, low-carbon travel } \\
\text { The Public Comments on Sharing bikes issued by Shanghai municipal government } \\
\text { indicated that 'Sharing bikes are usually scrapped after three years of use, and the } \\
\text { scrapped ones are not allowed to re-enter the market after repair and reassembly.' }\end{array}$ \\
\hline & Enabling technology (S1-P3-18) & Digital bicycle locks, GPS devices, 4G/5G network, the popularity of smartphones \\
\hline $\begin{array}{l}\text { Design concept } \\
\text { generation (S2- } \\
\text { P1) }\end{array}$ & Sketches with annotation (S2-P1-1) & \\
\hline \multirow{2}{*}{$\begin{array}{l}\text { Concept } \\
\text { development \& } \\
\text { evaluation } \\
\text { (S2-P2) }\end{array}$} & Product structure (S2-P2-8) & $\begin{array}{l}\text { Adjustable seat, airy basket, one sided frame, non-inflatable tires, frame, front wheel, rear } \\
\text { wheel, sprocket-pedal, chain, handlebar, hand brake, and suspension, etc. }\end{array}$ \\
\hline & Mechanisms (S2-P2-9) & $\begin{array}{l}\text { The lock system removed buttons altogether. The lock opens automatically once } \\
\text { authenticated. }\end{array}$ \\
\hline $\begin{array}{l}\text { Detail design and } \\
\text { evaluation } \\
\text { (S2-P3) }\end{array}$ & $\begin{array}{l}\text { Complete specification of the geometry, } \\
\text { material, and tolerances of all parts } \\
\text { (S2-P3-1) }\end{array}$ & 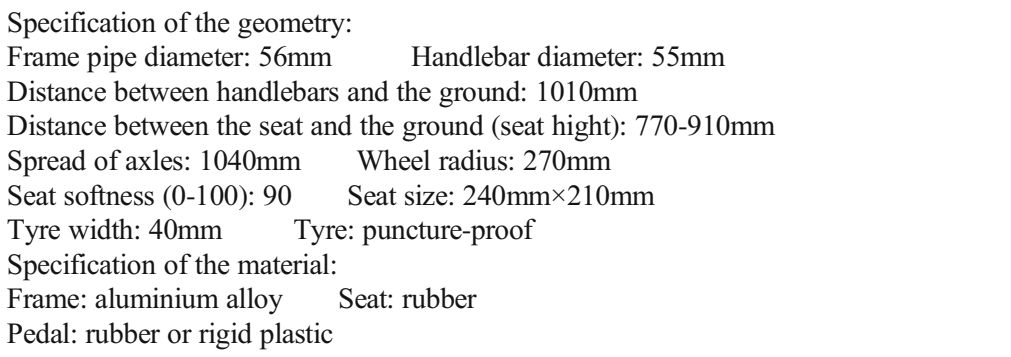 \\
\hline
\end{tabular}

CAx models (S2-P3-6)

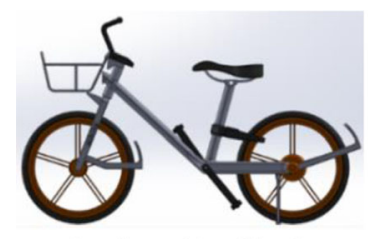

Bevel gear-driven bicycle

Detail and assembly drawings (S2-P32)

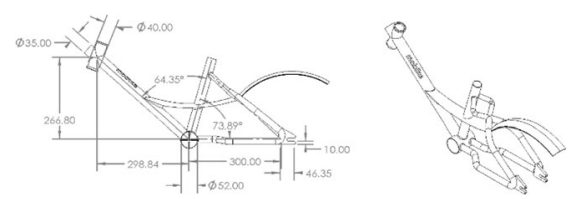

Engineering drawings of bicycle frame
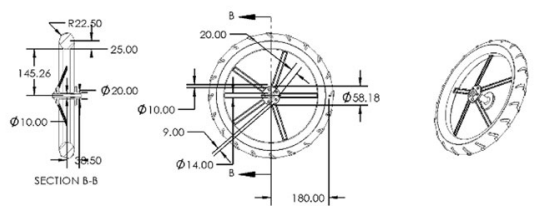
Table 4 (continued)

\section{Design phase \\ Key information items and their \\ Information values/representation examples}

codes

Parts list with raw material sizes and specifications (S2-P3-3)

Bill of materials (S2-P3-4)

Selection of materials (S2-P3-5)

\begin{tabular}{|c|c|c|c|}
\hline Parts & Material & Colour & Technical specification \\
\hline $\begin{array}{l}\text { Bicycle } \\
\text { frame }\end{array}$ & Aluminium alloy & Silver & V-shaped chassis \\
\hline Tyre & $\begin{array}{l}\text { Inner : Aluminium } \\
\text { alloy } \\
\text { Outer: Rubber }\end{array}$ & $\begin{array}{l}\text { Inner: silver } \\
\text { Outer: orange }\end{array}$ & $\begin{array}{l}\text { Wheel radius: } 270 \mathrm{~mm} \\
\text { Tire width: } 40 \mathrm{~mm} \\
\text { Tubeless tyres } \\
\text { Chain-driven model: } \\
\text { With spokes } \\
\text { Bevel gear-driven model: } \\
\text { With five sets of two thick, } \\
\text { parallel, metal rods positioned at } \\
72^{\circ} \text { from each other }\end{array}$ \\
\hline Seat & Rubber & Black & $\begin{array}{l}\text { Seat softness }(0-100): 90 \\
\text { Seat size: } 240 \mathrm{~mm} \times 210 \mathrm{~mm}\end{array}$ \\
\hline Chain & Manganese steel & Silver & $\begin{array}{l}\text { Chain-driven model: } \\
\text { Chain inner width: } 3.175 \mathrm{~mm} \\
\text { Chain outer width: } 5.25 \mathrm{~mm} \\
\text { Bevel gear-driven model: no }\end{array}$ \\
\hline Sprocket & Carbon stell & Silver & $\begin{array}{l}\text { Chain-driven model: } \\
\text { Number of teeth: } 38 \\
\text { Pitch Diameter: } 10 \mathrm{~mm} \\
\text { Outside diameter: } 151 \mathrm{~mm} \\
\text { Bevel gear-driven model: no }\end{array}$ \\
\hline pedal & $\begin{array}{l}\text { Rubber or rigid } \\
\text { plastic }\end{array}$ & Black & $100 \mathrm{~mm} \times 80 \mathrm{~mm}$ \\
\hline
\end{tabular}

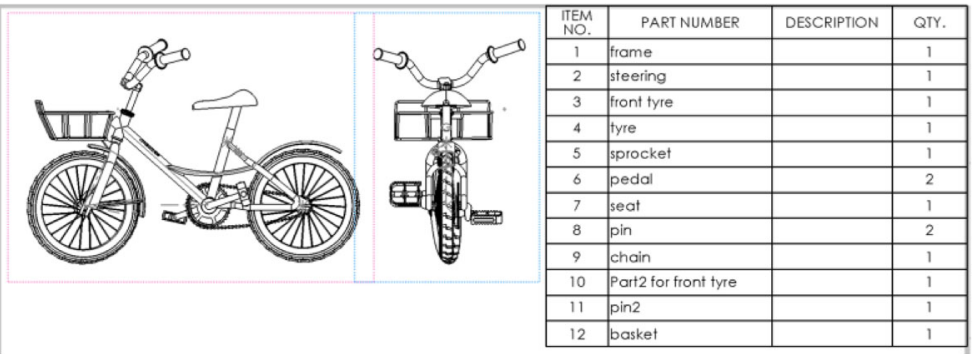

\begin{tabular}{|c|c|c|c|c|}
\hline & $\begin{array}{c}\text { Elastic Modulus } \\
\text { (GPa) }\end{array}$ & $\begin{array}{c}\text { Tensile Strength } \\
\text { (MPa) } \\
\end{array}$ & $\begin{array}{c}\text { Yield Strength } \\
\text { (MPa) }\end{array}$ & $\begin{array}{c}\text { Mass Density } \\
\left(\mathrm{kg} / \mathrm{m}^{\wedge} 3\right)\end{array}$ \\
\hline $\begin{array}{l}\text { Steels } \\
\text { AISI } 4130 \text { Steel } \\
\text { Alloy Steel }\end{array}$ & $\begin{array}{l}205 \\
210\end{array}$ & $\begin{array}{c}731 \\
723.83\end{array}$ & $\begin{array}{c}460 \\
620.42\end{array}$ & $\begin{array}{l}7850 \\
7700\end{array}$ \\
\hline $\begin{array}{r}\text { Aluminum Alloys } \\
2024-\mathrm{T} 4 \\
6061-\mathrm{T} 6 \\
7075-\mathrm{T} 6(\mathrm{SN})\end{array}$ & $\begin{array}{c}72.4 \\
69 \\
72 \\
\end{array}$ & $\begin{array}{l}470 \\
310 \\
570 \\
\end{array}$ & $\begin{array}{l}325 \\
275 \\
505\end{array}$ & $\begin{array}{l}2780 \\
2700 \\
2810 \\
\end{array}$ \\
\hline Titanium & 110 & 235 & 140 & 4600 \\
\hline Rubber & 0.0061 & 13.79 & 9.24 & 1000 \\
\hline $\begin{array}{r}\text { Plastics } \\
\text { Acrylic } \\
\text { Delrin 2700 NC010 } \\
\text { Nylon 6/10 }\end{array}$ & $\begin{array}{c}3 \\
2.9 \\
8.3\end{array}$ & $\begin{array}{c}73 \\
40.7 \\
142.56\end{array}$ & $\begin{array}{c}45 \\
63 \\
139.04\end{array}$ & $\begin{array}{l}1200 \\
1410 \\
1400\end{array}$ \\
\hline
\end{tabular}


Table 4 (continued)

\section{Design phase \\ Key information items and their} codes

Integrated service development (S2-P4)
Analysis results of the robustness \& performance of components and assemblies (S2-P3-9)
Service information flow (S2-P4-5)

Interactive touchpoints (S2-P4-6)

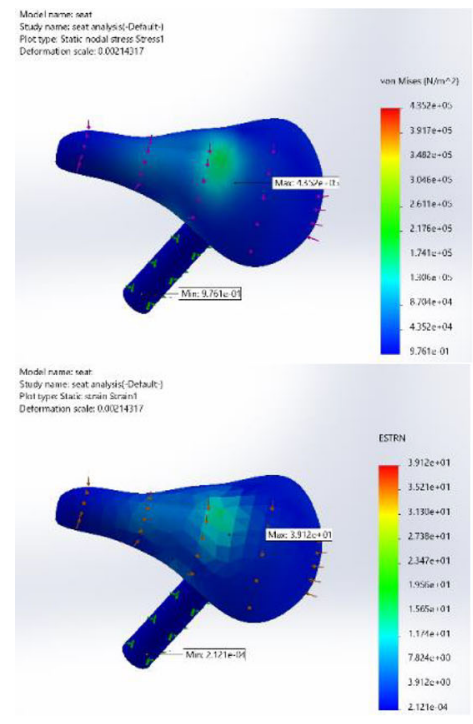

DFMEA of bicycle hand brake sub-system [54]:

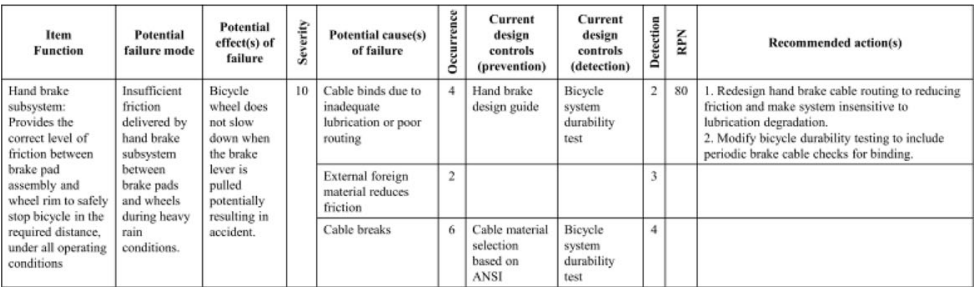

PFMEA of spokes assembly in a bicycle wheel [54]:

\begin{tabular}{|c|c|c|c|c|c|c|c|c|c|c|}
\hline $\begin{array}{l}\text { Process } \\
\text { function }\end{array}$ & $\begin{array}{c}\text { Potential } \\
\text { failure } \\
\text { mode }\end{array}$ & Potential effect(s) of failure & 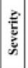 & $\begin{array}{l}\text { Potential } \\
\text { causefs) of } \\
\text { failure }\end{array}$ & & $\begin{array}{c}\text { Current } \\
\text { design controls } \\
\text { (prevention) }\end{array}$ & $\begin{array}{c}\text { Current design } \\
\text { controls } \\
\text { (detection) }\end{array}$ & & $\frac{t}{\underline{a}}$ & Recommended action(s) \\
\hline \multirow{2}{*}{$\begin{array}{l}\text { Orient } \\
\text { and place } \\
\text { whecl } \\
\text { spokes } \\
\text { properly } \\
\text { in wheel } \\
\text { assembly } \\
\text { fixture }\end{array}$} & $\begin{array}{l}\text { Too few } \\
\text { spokes }\end{array}$ & 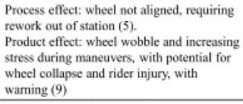 & 9 & 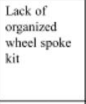 & 3 & $\begin{array}{l}\text { Wheel spoke } \\
\text { installation } \\
\text { vork } \\
\text { instructions }\end{array}$ & $\begin{array}{l}\text { Visual check of } \\
\text { wheel assembly } \\
\text { by opecrutor }\end{array}$ & 5 & 135 & $\begin{array}{l}\text { 1.Kit spokes into pre-defined } \\
\text { quantitics } \\
\text { 2.Develop and implement in- } \\
\text { station vision syptem to } \\
\text { detect missing whecl spokes }\end{array}$ \\
\hline & $\begin{array}{l}\text { Whect } \\
\text { spokes not } \\
\text { in nowrest } \\
\text { orientation }\end{array}$ & 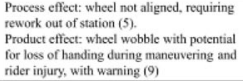 & 9 & 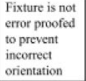 & 6 & $\begin{array}{l}\text { Whece spoke } \\
\text { installation } \\
\text { work } \\
\text { instructions }\end{array}$ & $\begin{array}{l}\text { In-station test for } \\
\text { wheel } \\
\text { alignment truing }\end{array}$ & 7 & & \\
\hline
\end{tabular}

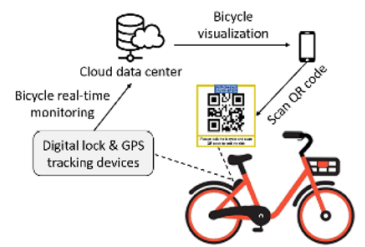

Service plan (S2-P4-13)

\begin{tabular}{|c|l|}
\hline Service plan & \multicolumn{1}{|c|}{ Price } \\
\hline Pay as you go & $\begin{array}{l}1.5 \mathrm{CNY} \text { for the first 15 minutes, and 1 CNY } \\
\text { for every 15 minutes thereafter. }\end{array}$ \\
\hline $\begin{array}{c}\text { Pay monthly with } \\
\text { unlimited riding }\end{array}$ & $20 \mathrm{CNY}$ \\
\hline $\begin{array}{c}\text { Pay monthly with } \\
\text { eight riding }\end{array}$ & $8 \mathrm{CNY}$ \\
\hline $\begin{array}{c}\text { Pay monthly with } \\
\text { subscription }\end{array}$ & $\begin{array}{l}20 \mathrm{CNY} \text { automatic renewal for 14.9 CNY per } \\
\text { month and can be cancelled at anytime }\end{array}$ \\
\hline
\end{tabular}


Table 4 (continued)

\begin{tabular}{|c|c|c|}
\hline Design phase & $\begin{array}{l}\text { Key information items and their } \\
\text { codes }\end{array}$ & Information values/representation examples \\
\hline \multirow[t]{2}{*}{$\begin{array}{l}\text { Manufacturing } \\
\text { scheduling } \\
\text { (S3-P1) }\end{array}$} & Cost of raw materials (S3-P1-2) & $\begin{array}{l}\text { The cost of a first generation Mobike is } 6,000 \mathrm{CNY} \text {, including whole aluminum frame, } \\
\text { non-inflatable rubber tires, and GPS-based smart lock, etc. The cost of a second } \\
\text { generation Mobike is decreased to under } 1,000 \mathrm{CNY} \text {. }\end{array}$ \\
\hline & Control plan (S3-P1-13) & Manufacturing aim: produce 14,000 Mobikes every day. \\
\hline $\begin{array}{l}\text { Actual parts } \\
\text { manufacturing } \\
(\mathrm{S} 3-\mathrm{P} 2)\end{array}$ & Changes to components/parts (S3-P2-4) & $\begin{array}{l}\text { Components added: Smart locks, GPS devices, SIM card, barcodes/QR codes. } \\
\text { Components deleted: key/card connected to payment, docking stations. }\end{array}$ \\
\hline $\begin{array}{l}\text { Product assembly } \\
\quad \text { (S3-P3) }\end{array}$ & $\begin{array}{l}\text { Assembly cost (S3-P3-1) } \\
\text { Assembly process (S3-P3-3) }\end{array}$ & $\begin{array}{l}\text { The changes to bike structure and the materials can lead to the change of assembly cost } \\
\text { and process. }\end{array}$ \\
\hline $\begin{array}{l}\text { Product testing } \\
\quad \text { (S3-P4) }\end{array}$ & $\begin{array}{l}\text { Product safety (S3-P4-3) } \\
\text { Product reliability (S3-P4-5) }\end{array}$ & $\begin{array}{l}\text { When designing the Mobike frame, tens of thousands of crash tests were carried out on } \\
\text { the Mobike to make it more reliable. }\end{array}$ \\
\hline $\begin{array}{l}\text { Product } \\
\text { transportation } \\
\text { (S3-P5) }\end{array}$ & Transportation cost (S3-P5-3) & $\begin{array}{l}\text { Li Gang, the founder of another sharing bike 'bluegogo' said: 'the transportation cost of a } \\
\text { scrapped bike is about } 80 \text { CNY.' }\end{array}$ \\
\hline $\begin{array}{l}\text { Product launch } \\
\quad \text { (S4-P1) }\end{array}$ & $\begin{array}{l}\text { Launch date (S4-P1-1) } \\
\text { Product promotion channels (S4-P1-2) }\end{array}$ & Mobike was first launched in Shanghai in April 2016. \\
\hline \multirow[t]{3}{*}{$\begin{array}{l}\text { Product in use } \\
\quad(\mathrm{S} 4-\mathrm{P} 2)\end{array}$} & Feedback from end users (S4-P2-3) & $\begin{array}{l}\text { Positive: improved users' experience at the end of their rides and flexible route and } \\
\text { destination choices. } \\
\text { Negative: a deposit around } 300 \mathrm{CNY} \text { is required before use, which is not friendly to } \\
\text { short-term city residents. }\end{array}$ \\
\hline & $\begin{array}{l}\text { Tools for collecting product-generated } \\
\text { and user-generated data (S4-P2-5) }\end{array}$ & $\begin{array}{l}\text {-the QR code is used to connect the dockless bicycle to the cloud data center. The users } \\
\text { can provide feedback to the product as well by scanning the QR code. } \\
\text {-Collect and display user's cycling data from GPS devices }\end{array}$ \\
\hline & Real-time data analysis tool (S4-P2-6) & $\begin{array}{l}\text { The server side of the dockless bicycle system provides online services such as real-time } \\
\text { data analysis, the management of all dockless bicycles, the monitor of bicycle status, } \\
\text { the payment for using the bicycle, etc. }\end{array}$ \\
\hline $\begin{array}{l}\text { Product } \\
\text { maintenance } \\
(\mathrm{S} 5-\mathrm{P} 1)\end{array}$ & $\begin{array}{l}\text { Maintenance cost (S5-P1-5) } \\
\text { Maintenance time (S5-P1-9) }\end{array}$ & $\begin{array}{l}\text {-The exceeding supply of dockless bike-sharing systems and inadequate redistribution } \\
\text { schemes led to a large amount of abandoned or damaged bikes remaining on the streets } \\
\text { without timely maintenance or clearance. } \\
\text {-The redistribution of shared bikes occurs on a larger geographic scale } \\
\text {-Controlling and regulating shared bikes becomes more difficult in regions that have } \\
\text { dockless systems }\end{array}$ \\
\hline \multirow[t]{2}{*}{$\begin{array}{l}\text { Product recycling } \\
\quad(\mathrm{S} 5-\mathrm{P} 2)\end{array}$} & $\begin{array}{l}\text { The reusable state (S5-P2-2) } \\
\text { Remaining service time of } \\
\text { parts/components (S5-P2-3) }\end{array}$ & $\begin{array}{l}\text { Mobike } ® \text { takes the recyclable parts of scrapped bikes for reuse or making other products. } \\
\text { For example, smart locks, solar panels and tyres will be tested and reused. } \\
\text { Unrecyclable parts mainly some mental parts and a small amount of plastic and rubber } \\
\text { that cannot be recycled by the supplier will be disposed by recycling companies. }\end{array}$ \\
\hline & $\begin{array}{l}\text { Percentage of recycled products } \\
\text { (S5-P2-9) }\end{array}$ & $\begin{array}{l}\text { According to Mobike® report, more than } 60,000 \text { scrapped Mobikes have been recycled } \\
\text { for protecting environment since April } 2018 \text {. }\end{array}$ \\
\hline
\end{tabular}


the design information in different product generations. Although the information values required for product design vary from product to product in a product family, the key information items (classified fields) at the higher/class level are roughly the same.

\section{Modelling information interaction in the PDLIM}

The first section demonstrates the generic product design perspective model (Fig. 10) and its mapping with the proposed PDLIM model (Fig. 11) to approve the PDLIM is useful for supporting product design information need at the framework level. The second section aims to demonstrate and qualitatively evaluate the secondary interaction information on the "Interaction" layer with object-role modelling (ORM) models [55] in exemplar application scenarios. Existing studies have identified that ORM is more suitable for formulating, transforming, or evolving a conceptual information model by using natural language, intuitive diagrams, and examples [55]. Therefore, ORM modelling method is adopted to describe how the identified information items are used and interacted by key role players in typical practices. The first application scenario is for demonstrating design information interactions between two different design phases (Fig. 12), the second illustrates the interaction model of stakeholders in a crowdsourcing process (Fig. 13), and the third gives information interaction between two different product design generations (Fig. 14). Before illustrating information interactions, a generic perspective model for product design representation and how it is mapped with the identified information (drawers) in the proposed PDLIM are provided.

\subsection{Design representation model and its mapping with PDLIM}

At each design phase identified in Fig. 3, to help a design team effectively communicate or "sell" their design ideas or results at different stages to funders, partners, consumers and other stakeholders, information in the PDLIM needs to be synthesized together to produce a good design representation. Design representations can be made before, during, and after the process of designing any entity, regardless of whether the designed entity is being constructed, manufactured, and assembled as a real product [56].

Goldschmidt [56] considers that design representation can be used to convey messages concerning a wide cultural, social, economic, and technological context in which the design has been conceived and is to be integrated. In order to identify the required information for a product design representation, Riesener et al. [48] proposed a generic perspective model that combines eight different perspectives for describing a product design, as shown in Fig. 10.

The generic perspective model in Fig. 10 does not conflict with the existing design methodology. The requirements, functional, product structural, and production perspectives can be mapped to the four domains namely customer (CAs), functional (FRs), physical (DPs), and process (PVs) domain in axiomatic design framework [57]. The other perspectives can be described as constraints (Cs).

Taking the product design generic perspective model as the basis, the identified key information perspectives (drawers in Fig. 3) in the proposed PDLIM can be mapped to these eight perspectives under the Axiomatic Design framework (see Fig. 11). With changes occurring in the PDLIM drawer over time, the corresponding

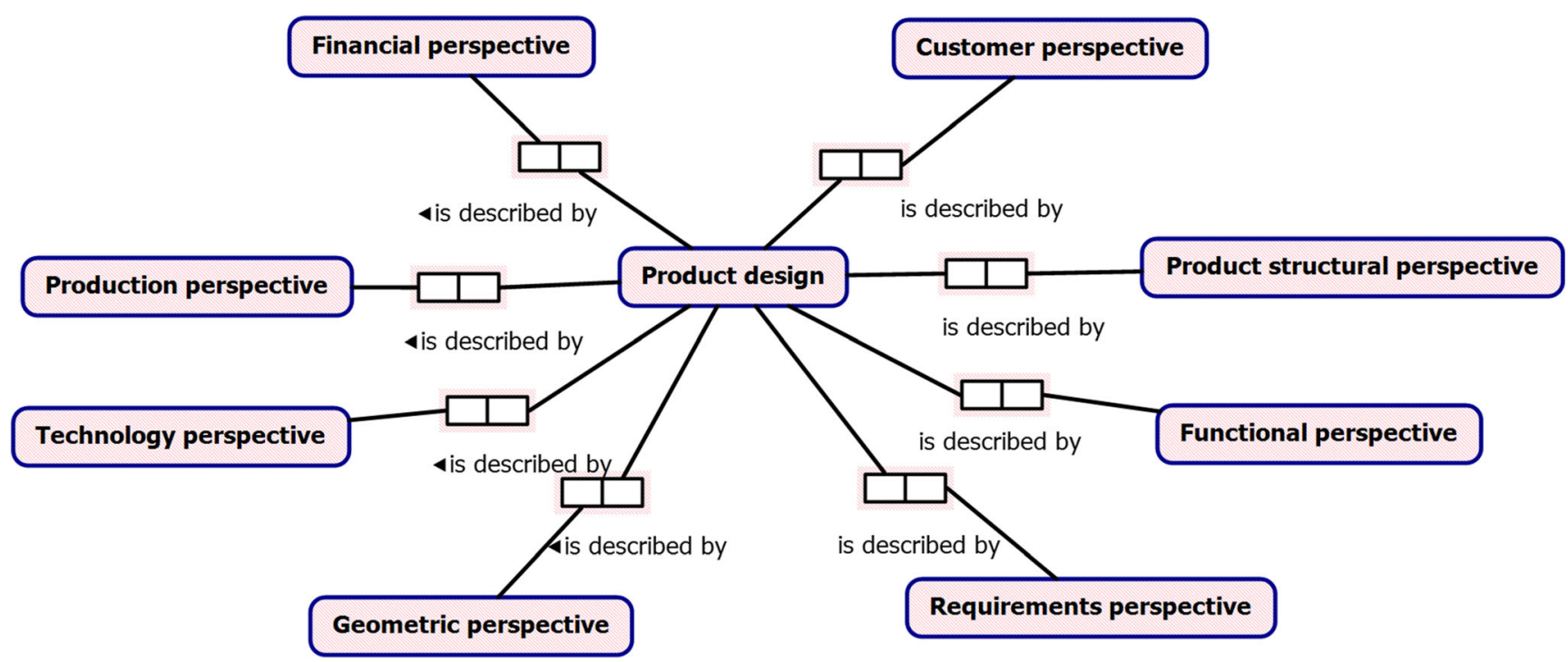

Fig. 10 Generic perspective model for product design representation 


\section{Drawer in PDLIM}

Generic perspective model

Market needs analysis (S1-P1)

User needs analysis (S1-P2)

Product design specification (S1-P3)

Needs evaluation \& review (S1-P4) (S3-P2)

Design concept generation (S2-P1)

Concept development \& evaluation (S2-P2)

Detail design and evaluation (S2-P3)

Integrated service development (S2-P4)

Manufacturing scheduling (S3-P1)

Actual product parts manufacturing (S3-P2)

Product assembly (S3-P3)

Product testing and evaluation (S3-P4)

Product transportation (S3-P5)

Product launch (S4-P1)

Product in use (S4-P2)

Product maintenance (S5-P1)

Product recycling (S5-P2)

In-house environment (E1-F1 to E1-F3)

Crowdsourcing environment (E2-F1 to E2-F6)

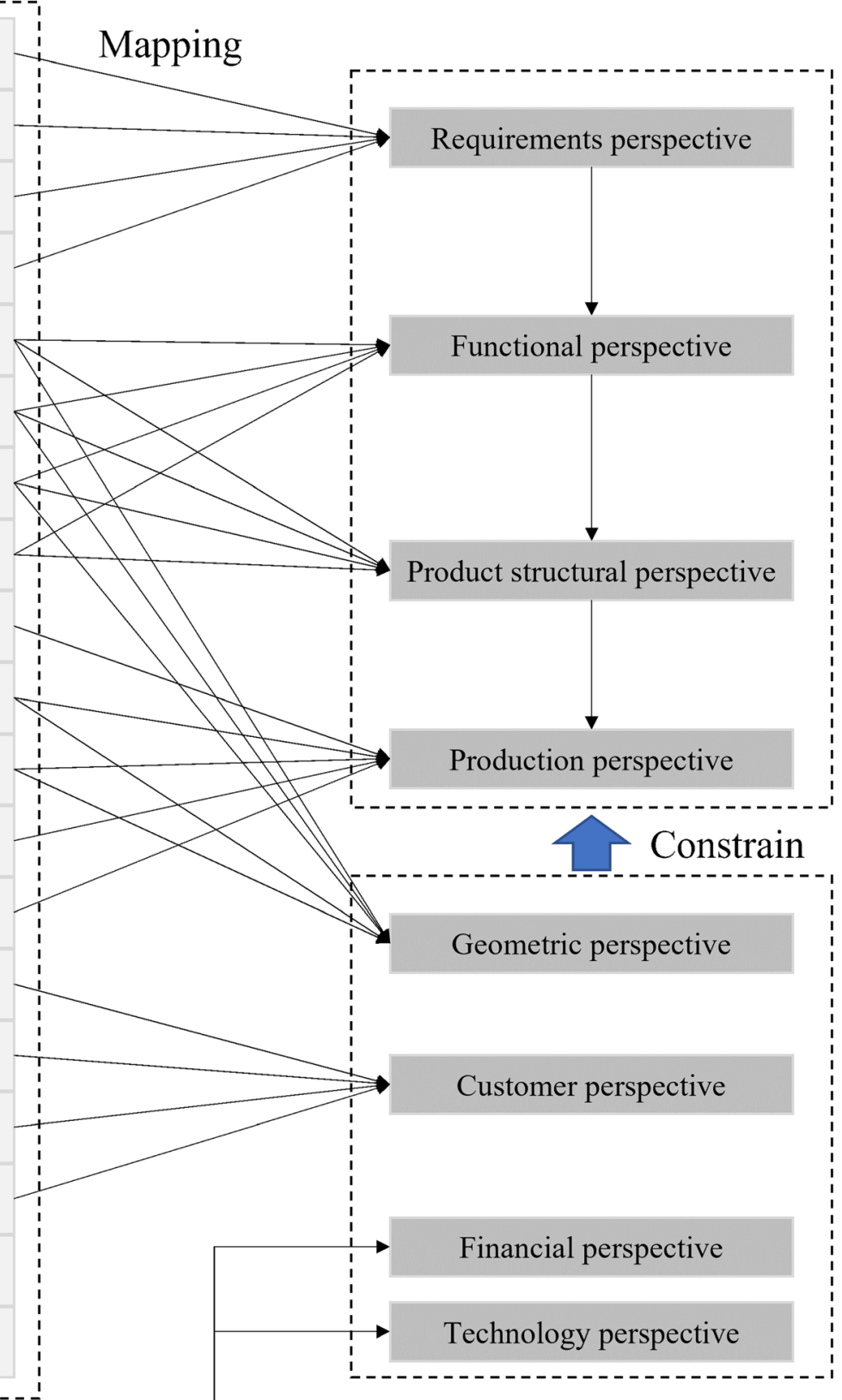

Fig. 11 The mapping between the key information perspectives in PDLIM and the product design generic perspective model

perspective would be changed as well, leading to the evolution of the product design. This conceptually proves that the PDLIM can support the information need for product (family) design. In this process, the information quality affects the product design quality. We are not discussing the information quality control here because there are many existing research works on how to measure information quality. For example, Riesener et al. [48] adopted accuracy, completeness, timeliness, comprehensibility, and consistency to describe data quality.

\subsection{Information interaction models in typical scenarios}

To understand how the key design information items identified in Fig. 4 to Fig. 8 are used and interacted with other information items, three typical application scenarios involving the interactions between two different design phases, among stakeholders in a crowdsourcing PDD process, and between two Mobike generations are modeled in Figs. 12, 13 , and 14 , respectively. 


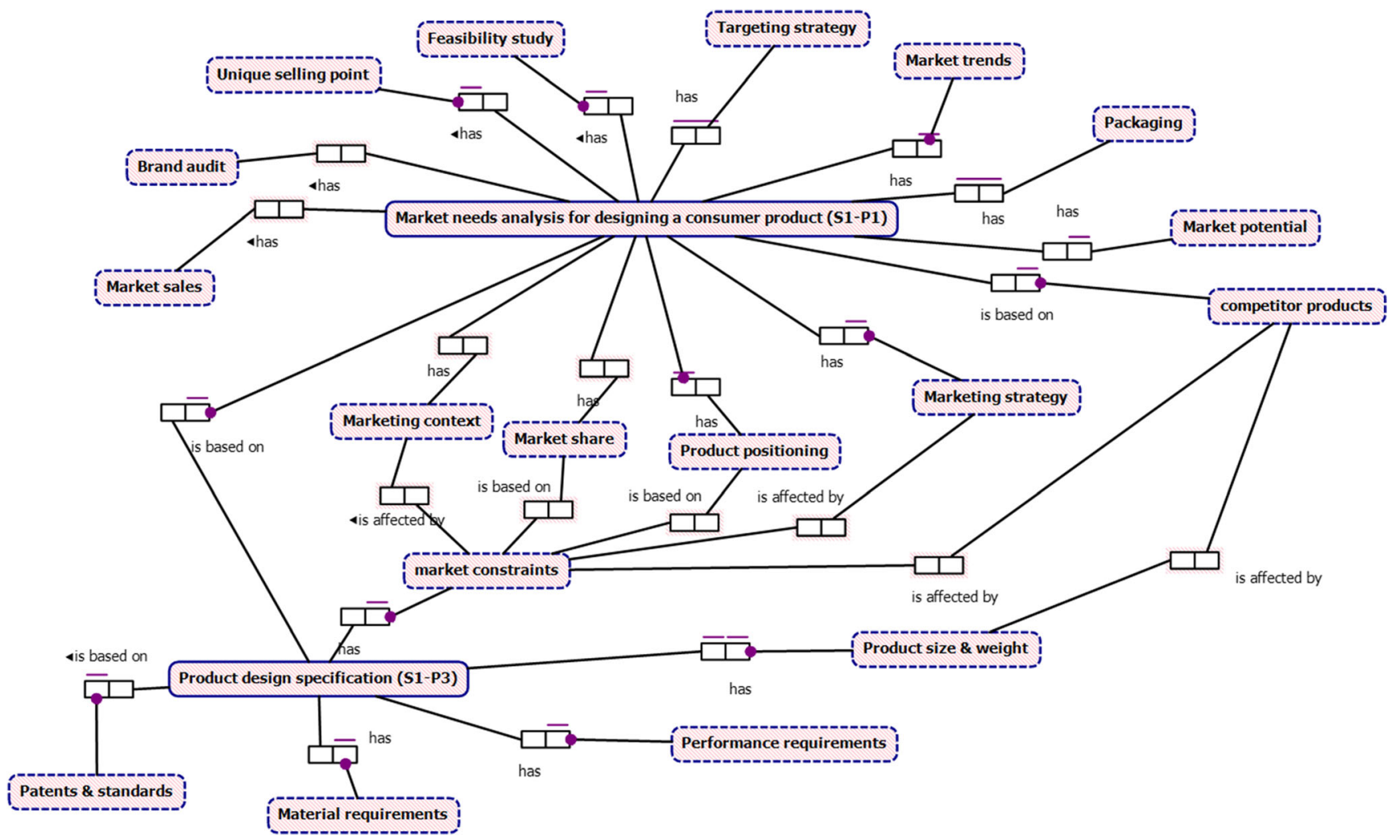

Fig. 12 An exemplar ORM model showing information interaction among different design phases

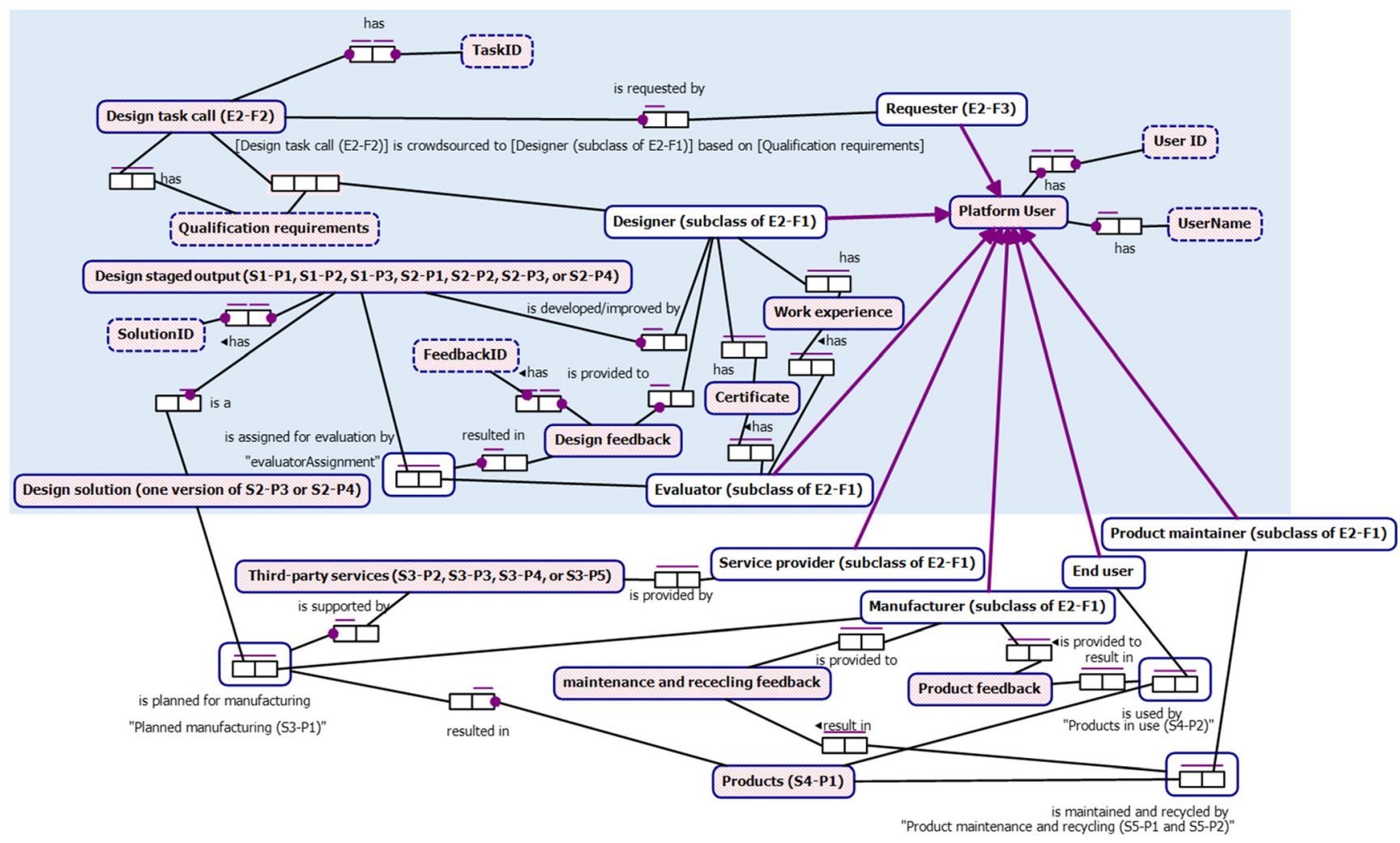

Fig. 13 ORM interaction model of relationships among identified entities in a crowdsourcing PDD process 


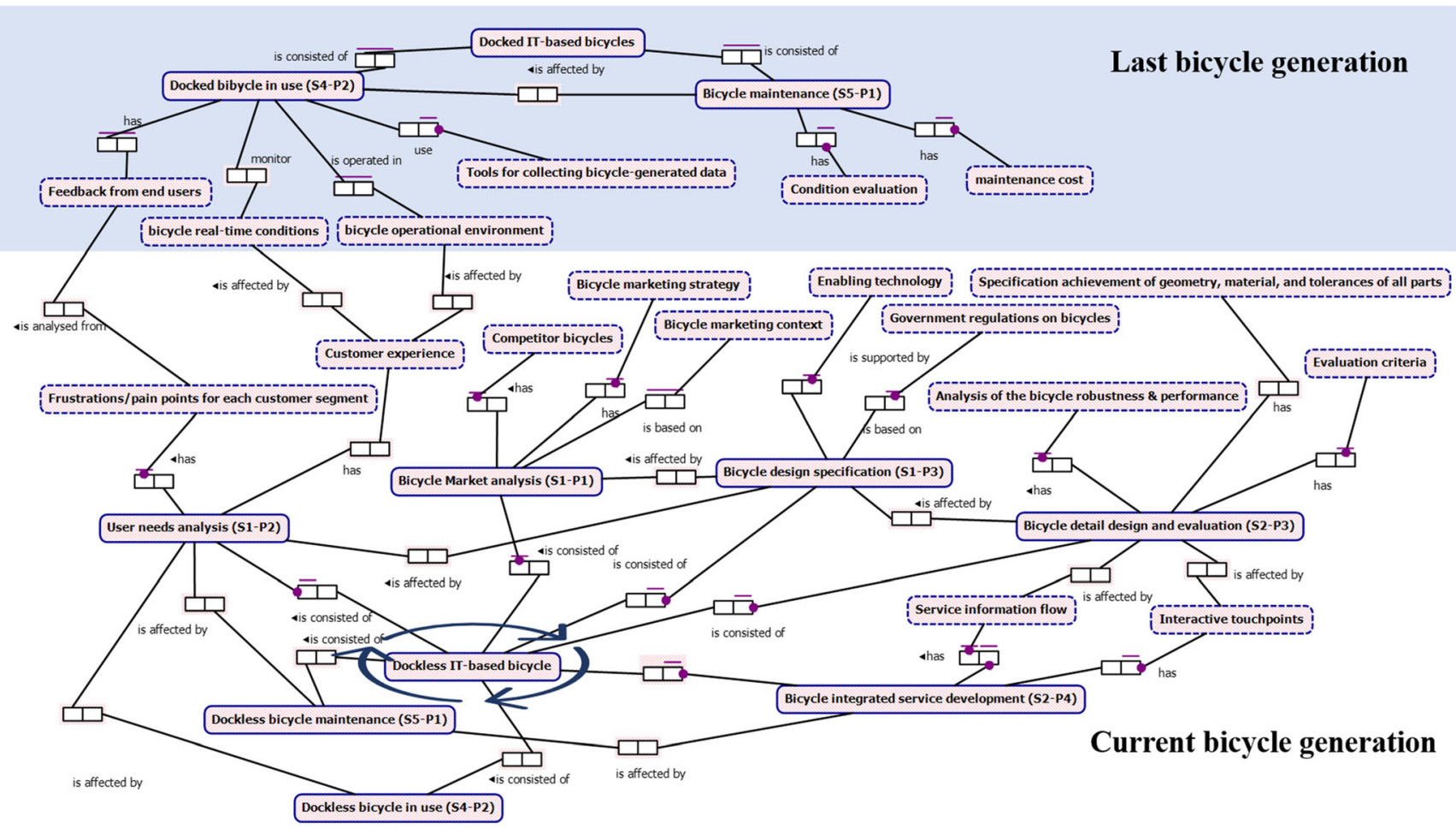

Fig. 14 Information relationship between two bicycle generations

(1) Interaction model between two design phases

Between different design phases, the later one can use information from the previous one. An ORM model in Fig. 12 indicates the information interaction between market needs analysis (S1-P1) and product design specification (S1-P3) phases and how the identified information in S1-P1 is used in S1-P3 phase. The ORM model mainly focuses on the information items (modelled as ORM entities) communicated between these two design phases.

\section{(2) Interaction model among stakeholders in a crowdsourcing PDD process}

The design environment information especially in the crowdsourcing environment is modelled in an ORM model to help evaluate and illustrate how to share and communicate the collected information items among platform users throughout a crowdsourcing PDD process.

All information items that are relevant and meaningful to the crowdsourcing PDD process, such as key role players, design task call, design staged output, and design feedback, are modelled as ORM object entities. Then an ORM model (Fig. 13) showing relationships among them in a crowdsourcing PDD process has been developed which only modelled entity ID as key entity identifier. Work experience and certificate are key indicators to measure the qualification of a designer or an evaluator. To record more details about the qualification instead of statistical data like most existing crowdsourcing systems, work experience and certificate are treated as entity types rather than value types.

In the model, all role players involved in the crowdsourcing PDD process are treated as platform users. Requester, designer, evaluator, manufacturer, service provider, and end user are key role players inherited from platform user in a crowdsourcing PDD process. At the product design stage, the key involved role players are requester, designer, and evaluator. Here, a requester refers to an individual project leader or an organization that launches the product design call/task, a designer refers to the crowd that can provide design ideas and solutions, and an evaluator refers to the crowd that can assess design ideas/solutions or business organizations that can provide design assessment services.

The relationship ORM model has the following features:

- It illustrates the design process and key interactions among platform users when crowdsourcing a product design task. The crowdsourcing PDD process starts from the request of a design task call, then it is crowdsourced to design professionals or general crowd designers by matching the participants' requirements in the design task call from the crowd designers' profiles. When the design task is accepted by a crowd designer, the designer will iteratively 
work on the design solutions with feedback from design evaluators until the solutions are satisfactory. In this process, all identified entities have their unique identifier.

- It is extendable. Before a design solution is ready for manufacturing, the key involved role players are the requester, designers, and evaluators, and their interactions are modelled as light blue area in Fig. 13. But when it comes to the whole PDD process, more role players, such as a manufacturer, service providers, and end users will be involved, and their interactions with existing identified entities can be easily modelled as shown in Fig. 13. In this process, when the manufacturer receives product feedback from end users, it can determine when to start a new round PDD process to improve its products.

With the ORM model, the information created and owned by a role player can be shared with others, making them aware of the whole product design progress and in turn informing their decision makings. Meanwhile, with feedback from other role players, more insightful suggestions for next actions can be obtained.

\section{(3) Interaction model between two Mobike generations}

To demonstrate how to use the PDLIM to establish the information interaction relationship between products in different generations, the second case study 'the design of dockless sharing bikes' in Section 4.2 is taken as an example. The corresponding ORM model is shown in Fig. 14. The light blue area shows key information items from docked bicycles in their use and maintenance phases, while the remaining part shows key information items used for designing current generational dockless bicycles. The information items from both the previous generation and current generation bicycles have an impact on designing a product in a product family within the same bicycle generation. In the current dockless bicycle generation, the design process is generative. With the PDLIM, the designer and his/her collaborators can track the design information changes easily.

With the PDLIM, it is easy to record any design changes/versions in the whole product design process, making it easier for designers and their collaborators in tracing and understanding the design information changes and decisions made. In addition, the PDLIM could establish a unified design information wardrobe structure for structurally classifying, capturing, storing, and sharing key information in an iterative product design and development process, thus bettering design information management, and supporting data-informed higher design quality and decision-making.

\section{Conclusion and future work}

This paper proposed a new PDLIM enabling product throughlife design and development trends under an in-house and crowdsourcing combined design environment. Firstly, a multilayered product design lifecycle information modelling structure is developed to accommodate all design related information. Secondly, the key information items at each design phase associated with both in-house and crowdsourcing design environments are identified to enrich the information model. Thirdly, the proposed PDLIM is qualitatively evaluated by two case studies. Finally, some typical interactions among the key information items and related key role players are modelled by ORM models to qualitatively evaluate the relationships and interactions of information entities in the PDLIM as well as to demonstrate the PDLIM's manageability.

From the above development and evaluations, we can conclude that the proposed PDLIM

(1) can serve PDD in either a traditional in-house design environment or a combined in-house and crowdsourcing design environment, making design opener with flexible crowd resource,

(2) has a great potential to be incorporated into a digital twinning platform, supporting the emerging datainformed product family development paradigm and smart manufacturing in a closed-loop PDD process,

(3) has a great potential to support effective design communication and information sharing and reuse during a product lifecycle development, bettering design quality and decision-making.

In the future, the research directions will concentrate on:

(1) Development of the lifecycle design information management system. To support data-informed decisionmaking in a PDD process, a design information management system for efficient information retrieval and analysis is a must. Based on that, data protection, processing, and big data analytics will be progressed.

(2) Data protection. Data protection is always a serious issue for any enterprise. To help manufacturers make decisions during their PDD process, the PDLIM may cover some sensitive business information such as intellectual property and business plans. Hence, new methods should be developed to balance information sharing and protection.

(3) Data processing. In the whole product lifecycle, a large volume of data with various formats will be generated and collected from different design phases. To further model and visualize these collected data, new data processing and analysis methods should be developed to transform them into easily understandable and explainable information formats. 
(4) Integration of DT with big data and machine learning technology for product design support. With the increase of design information in database, DT will be integrated with big data and machine learning technology to facilitate new levels of design understanding and to help designers make quick design decisions, thus helping manufacturers gain competitive time to market.

Acknowledgements Thanks are due to the designer student Kyle Richards in the first case study for providing his critical justifications (the documents to record his design process) and presentation documents for analysis.

Author contribution The first author is the $\mathrm{PhD}$ student contributing to the secondary and primary research. The second author is the collaborative supervisor on information management. The third author is the corresponding author, the $\mathrm{PhD}$ supervisor, contributing to the overall research planning and quality control.

Funding This research was supported by China Scholarship Council (Grant No. CSC.201606300041).

Data availability None

Declarations This paper is from our original research work; the work described has not been published before; it is not under consideration for publication anywhere else; and its publication has been approved by all co-authors.

Ethics approval This research is based on a jointly funded $\mathrm{PhD}$ research work. It has its ethical approval from Northumbria University.

Consent to participate The researchers have informed consents from all participants.

Consent for publication All authors and participants gave their consent to publish.

Competing interests The authors declare no competing interests.

Open Access This article is licensed under a Creative Commons Attribution 4.0 International License, which permits use, sharing, adaptation, distribution and reproduction in any medium or format, as long as you give appropriate credit to the original author(s) and the source, provide a link to the Creative Commons licence, and indicate if changes were made. The images or other third party material in this article are included in the article's Creative Commons licence, unless indicated otherwise in a credit line to the material. If material is not included in the article's Creative Commons licence and your intended use is not permitted by statutory regulation or exceeds the permitted use, you will need to obtain permission directly from the copyright holder. To view a copy of this licence, visit http://creativecommons.org/licenses/by/4.0/.

\section{References}

1. Zhang H, Qin S, Li R, Zou Y, Ding G (2019) Progressive modelling of feature-centred product family development. Int J Prod Res 58(12):3701-3723. https://doi.org/10.1080/00207543.2019. 1634295
2. Gauss L, Lacerda D, Miguel P (2021) Module-based product family design: systematic literature review and meta-synthesis. J Intell Manuf 32:265-312. https://doi.org/10.1007/s10845-020-01572-3

3. Li Q, Luo H, Xie P, Feng X, Du R (2015) Product whole life-cycle and omni-channels data convergence oriented enterprise networks integration in a sensing environment. Comput Ind 70:23-45. https://doi.org/10.1016/j.compind.2015.01.011

4. Tao F, Cheng J, Qi Q, Zhang M, Zhang H, Sui F (2018) Digital twin-driven product design, manufacturing and service with big data. Int J Adv Manuf Technol 94:3563-3576. https://doi.org/10. 1007/s00170-017-0233-1

5. Forbes H, Schaefer D (2018) Crowdsourcing in product development: current state and future research directions. The 15th International Design Conference Dubrovnik, Croatia, pp 579588. https://doi.org/10.21278/idc.2018.0161

6. Jiao Y, Wu Y, Lu S (2021) The role of crowdsourcing in product design: the moderating effect of user expertise and network connectivity. Technol Soc 64:101496. https://doi.org/10.1016/j.techsoc. 2020.101496

7. Niu X, Qin S, Zhang H, Wang M, Wong R (2018) Exploring product design quality control and assurance under both traditional and crowdsourcing-based design environments. Adv Mech Eng 10(12):168781401881439. https://doi.org/10.1177/ 1687814018814395

8. Florian D, Kucherbaev P, Cappiello C, Benatallah B, Allahbakhsh M (2018) Quality control in crowdsourcing: a survey of quality attributes, assessment techniques, and assurance actions. ACM Comput Surv 51(1):1-40. https://doi.org/10.1145/3148148

9. Schleich B, Anwer N, Mathieu L, Wartzack S (2017) Shaping the digital twin for design and production engineering. CIRP Ann 66(1):141-144. https://doi.org/10.1016/j.cirp.2017.04.040

10. Raj P (2021) Empowering digital twins with blockchain. Adv Comput 121:267-283. https://doi.org/10.1016/bs.adcom.2020.08.013

11. Roy R, Mishra D, Pal S, Chakravarty T, Panda S, Chandra M, Pal A, Misra P, Chakravarty D, Misra S (2020) Digital twin: Current scenario and a case study on a manufacturing process. Int J Adv Manuf Technol 107:3691-3714. https://doi.org/10.1007/s00170-020-05306-w

12. Tao F, Zhang H, Liu A, Nee A (2018) Digital twin in industry: state-of-the-art. IEEE Trans Ind Inform 15(4):2405-2415. https:// doi.org/10.1109/TII.2018.2873186

13. Jones D, Snider C, Kent L, Hicks B (2019) Early stage digital twins for early stage engineering design. International Conference on Engineering Design Delft, Netherlands, pp 2557-2566. https:// doi.org/10.1017/dsi.2019.262

14. Lim K, Zheng P, Chen C, Huang L (2020) A digital twin-enhanced system for engineering product family design and optimization. $\mathrm{J}$ Manuf Syst 57:82-93. https://doi.org/10.1016/j.jmsy.2020.08.011

15. Sudarsan R, Fenves SJ, Sriram RD, Wang F (2005) A product information modeling framework for product lifecycle management. Comput Aided Des 37(13):1399-1411. https://doi.org/10. 1016/j.cad.2005.02.010

16. Tao F, Sui F, Liu A, Qi Q, Zhang M, Song B, Guo Z, Lu S, Nee A (2019) Digital twin-driven product design framework. Int J Prod Res 57(12):3935-3953. https://doi.org/10.1080/00207543.2018.1443229

17. Choi J, Dyke S (2020) CrowdLIM: Crowdsourcing to enable lifecycle infrastructure management. Comput Ind 115:103185. https://doi.org/10.1016/j.compind.2019.103185

18. Kerin M, Pham D (2019) A review of emerging industry 4.0 technologies in remanufacturing. J Clean. Prod 237:117805. https://doi. org/10.1016/j.jclepro.2019.117805

19. Kong T, Hu T, Zhou T, Ye Y (2021) Data construction method for the applications of workshop digital twin system. J Manuf Syst 58: 323-328. https://doi.org/10.1016/j.jmsy.2020.02.003

20. Jauregui-Becker J, Wits W (2013) An information model for product development: a case study at PHILIPS Shavers. Procedia CIRP 9:97-102. https://doi.org/10.1016/j.procir.2013.06.175 
21. Li Z, Ramani K (2007) Ontology-based design information extraction and retrieval. Artif Intell Eng Des Anal Manuf 21(2):137-154. https://doi.org/10.1017/S0890060407070199

22. Li Z, Zhou X, Wang W, Huang G, Tian Z, Huang S (2018) An ontology-based product design framework for manufacturability verification and knowledge reuse. Int J Adv Manuf Technol 99(912):2121-2135. https://doi.org/10.1007/s00170-018-2099-2

23. Song X, Chai X, Zhang L, Gong G (2010) Modeling framework for product lifecycle information. Simul Model Pract Th 18(8):1080 1091. https://doi.org/10.1016/j.simpat.2009.07.004

24. Wright I (1998) Design methods in engineering and product design. McGraw-Hill

25. Zhu Y, Alard R, You J, Schönsleben P (2011) Collaboration in the design-manufacturing chain: a key to improve product quality. In: Renko S (ed) Supply Chain Management-New Perspectives, IntechOpen. https://doi.org/10.5772/799

26. Benabdellah A, Bouhaddou I, Benghabrit A, Benghabrit O (2019) A systematic review of design for X techniques from 1980 to 2018: concepts, applications, and perspectives. Int J Adv Manuf Technol 102(9-12):3473-3502. https://doi.org/10.1007/s00170-019-03418-6

27. Liu D, Xu X (2001) A review of web-based product data management systems. Comput Ind 44(3):251-262. https://doi.org/10.1016/ S0166-3615(01)00072-0

28. Lee C, Leem C, Hwang I (2011) PDM and ERP integration methodology using digital manufacturing to support global manufacturing. Int J Adv Manuf Technol 53(1-4):399-409. https://doi.org/10. 1007/s00170-010-2833-x

29. Chodorow K (2013) MongoDB: the definitive guide: powerful and scalable data storage. O'Reilly Media Inc.

30. Pfeifer $M(2009)$ The materials engineering perspective to product design and manufacturing. In: Pfeifer M (ed) Materials enabled designs, 1st edn. Butterworth-Heinemann. https://doi.org/10.1016/ B978-0-7506-8287-9.X0001-8

31. Johnson A, Gibson A (2014) The tools of the design process and management of design. In: Johnson A, Gibson A (eds) Sustainability in engineering design. Academic Press, pp 113180. https://doi.org/10.1016/B978-0-08-099369-0.00004-2

32. Sanfilippo E, Borgo S (2016) What are features? An ontologybased review of the literature. Comput Aided Des 80:9-18. https://doi.org/10.1016/j.cad.2016.07.001

33. Eckert C, Wynn D, Maier J, Albers A, Bursac N, Xin C, Clarkson P, Gericke K, Gladysz B, Shapiro D (2017) On the integration of product and process models in engineering design. Des Sci 3. https://doi.org/10.1017/dsj.2017.2

34. Lee Y (1999) Information modeling: From design to implementation. The second world manufacturing congress: International Computer Science Conventions Durham, UK, pp 315-321

35. Rachuri S, Subrahmanian E, Bouras A, Fenves S, Foufou S, Sriram R (2008) Information sharing and exchange in the context of product lifecycle management: Role of standards. Comput Aided Des 40(7):789-800. https://doi.org/10.1016/j.cad.2007.06.012

36. Loos L, Verbeeck K, Laet L (2019) Data visualisation as a tool for informed structural design. Comput Aided Des 115:267-276. https://doi.org/10.1016/j.cad.2019.06.003

37. Kolich M (2014) Using failure mode and effects analysis to design a comfortable automotive driver seat. Appl Ergon 45(4):1087-1096. https://doi.org/10.1016/j.apergo.2014.01.007

38. Li J, Tao F, Cheng Y, Zhao L (2015) Big data in product lifecycle management. Int J Adv Manuf Technol 81:667-684. https://doi. org/10.1007/s00170-015-7151-x

39. Rajamani M, Punna E (2019) A framework for effective implementation of process failure modes and effects analysis with control plans to mitigate process, discrete manufacturing and service industries, using aerospace standard best practices. SAE Tech Pap 1-9. https://doi.org/10.4271/2019-28-0149

40. Wynn D, Clarkson P (2018) Process models in design and development. Res Eng Des 29(2):161-202. https://doi.org/10.1007/ s00163-017-0262-7

41. CeArley D, Burke B, Searle S, Walker M (2016) Top 10 strategic technology trends for 2018. https://www.gartner.com/en/doc/ 3811368-top-10-strategic-technology-trends-for-2018. Accessed 2020

42. Jiang H, Qin S, Fu J, Zhang J, Ding G (2021) How to model and implement connections between physical and virtual models for digital twin application. J Manuf Syst 58:36-51. https://doi.org/ 10.1016/j.jmsy.2020.05.012

43. Rasheed A, San O, Kvamsdal T (2020) Digital twin: Values, challenges and enablers from a modeling perspective. IEEE Access 8: 21980-22012. https://doi.org/10.1109/ACCESS.2020.2970143

44. Reyna A, Martín C, Chen J, Soler E, Díaz M (2018) On blockchain and its integration with IoT: Challenges and opportunities. Futur Gener Comput Syst 88:173-190. https://doi.org/10.1016/j.future. 2018.05.046

45. Bhatti S, Gao X, Chen G (2020) General framework, opportunities and challenges for crowdsourcing techniques: a Comprehensive survey. J Syst Softw 167:110611. https://doi.org/10.1016/j.jss. 2020.110611

46. Hetmank L (2013) Components and functions of crowdsourcing systems - a systematic literature review. The 11th International Conference on Wirtschaftsinformatik Leipzig, Germany, pp 5569. https://doi.org/10.13140/2.1.3836.4166

47. Cheng F, Yu S, Qin S, Chu J, Chen J (2021) User experience evaluation method based on online product reviews. J Intell Fuzzy Syst 41(1):1791-1805. https://doi.org/10.3233/JIFS-210564

48. Riesener M, Schuh G, Dölle C, Tönnesa C (2019) The digital shadow as enabler for data analytics in product life cycle management. Procedia CIRP 80:729-734. https://doi.org/10.1016/j.procir.2019. 01.083

49. Prince J (2014) An investigation into bicycle performance and design. Auckland University of Technology, Dissertation

50. Hadland T, Lessing H, Clayton N, Sanderson G (2014) Bicycle design: an illustrated history. The Mit Press, London

51. Wang J, Huang J, Dunford M (2019) Rethinking the utility of public bicycles: the development and challenges of station-less bike sharing in China. Sustainability 11(6):1539. https://doi.org/10. 3390/su11061539

52. Yang T, Li Y, Zhou S (2019) System dynamics modeling of dockless bike-sharing program operations: a case study of mobike in Beijing, China. Sustainability 11(6):1601. https://doi.org/10. 3390/su11061601

53. Chen Z, Lierop D, Ettema D (2020) Dockless bike-sharing systems: what are the implications? Transp Rev 40(3):333-353. https://doi. org $/ 10.1080 / 01441647.2019 .1710306$

54. Carlson C (2014) Understanding and applying the fundamentals of FMEAs. 2015 Annual Reliability and Maintainability Symposium 10:1-35

55. Terry H (2015) Object-role modeling fundamentals: a practical guide to data modeling with ORM. Technics Publications, UK

56. Goldschmidt G (2004) Design representation: private process, public image. In: Goldschmidt G, Porter W (eds) Design representation. Springer, London. https://doi.org/10.1007/978-1-85233-863-3 9

57. Suh N (1995) Axiomatic design of mechanical systems. J Mech Des 117(B):2-10. https://doi.org/10.1115/1.2836467

Publisher's note Springer Nature remains neutral with regard to jurisdictional claims in published maps and institutional affiliations. 